\title{
Cell and Nucleus Shape as an Indicator of Tissue Fluidity in Carcinoma
}

\author{
Steffen Grosser®, ${ }^{1, *}$ Jürgen Lippoldt๑, ${ }^{1, *}$ Linda Oswald, ${ }^{1, *}$ Matthias Merkel®, ${ }^{2}$ Daniel M. Sussman $\odot{ }^{3}$ \\ Frédéric Renner, ${ }^{1}$ Pablo Gottheil, ${ }^{1}$ Erik W. Morawetz $\odot,{ }^{1}$ Thomas Fuhs $\odot,{ }^{1}$ Xiaofan Xie, ${ }^{1}$ Steve Pawlizak, ${ }^{1}$ \\ Anatol W. Fritsch $\odot,{ }^{1}$ Benjamin Wolf $\odot,{ }^{4}$ Lars-Christian Horn $\odot,{ }^{4}$ Susanne Briest, ${ }^{5}$ Bahriye Aktas $\odot,{ }^{5}$ \\ M. Lisa Manning $\oplus^{3}$ and Josef A. Käs $\circledast^{1, \dagger}$ \\ ${ }^{1}$ Peter Debye Institute, Leipzig University, Linnéstraße 5, 04103 Leipzig, Germany \\ ${ }^{2}$ Aix Marseille Univ, Université de Toulon, CNRS, CPT, Turing Center for Living Systems, \\ 13009 Marseille, France \\ ${ }^{3}$ Department of Physics, Syracuse University, Syracuse, New York 13244, USA \\ ${ }^{4}$ Institute for Pathology, Leipzig University, 04103 Leipzig, Germany \\ ${ }^{5}$ Department of Gynecology, Leipzig University, 04103 Leipzig, Germany
}

(Received 28 October 2020; revised 21 December 2020; accepted 23 December 2020; published 17 February 2021)

\begin{abstract}
Tissue, cell, and nucleus morphology change during tumor progression. In 2D confluent cell cultures, different tissue states, such as fluid (unjammed) and solid (jammed), are correlated with cell shapes. These results do not have to apply a priori to three dimensions. Cancer cell motility requires and corresponds to a fluidization of the tumor tissue on the bulk level. Here, we investigate bulk tissue fluidity in 3D and determine how it correlates with cell and nucleus shape. In patient samples of mamma and cervix carcinoma, we find areas where cells can move or are immobile. We compare 3D cell spheroids composed of cells from a cancerous and a noncancerous cell line. Through bulk mechanical spheroid-fusion experiments and single live-cell tracking, we show that the cancerous sample is fluidized by active cells moving through the tissue. The healthy, epithelial sample with immobile cells behaves more solidlike. 3D segmentations of the samples show that the degree of tissue fluidity correlates with elongated cell and nucleus shapes. This correlation links cell shapes to cell motility and bulk mechanical behavior. We find two active states of matter in solid tumors: an amorphous glasslike state with characteristics of 3D cell jamming and a disordered fluid state. Individual cell and nucleus shape may serve as a marker for metastatic potential to foster personalized cancer treatment.
\end{abstract}

DOI: 10.1103/PhysRevX.11.011033

Subject Areas: Biological Physics

\section{INTRODUCTION}

In cancer progression, epithelial tissues develop into malignant tumors that are densely packed with cancer cells. Thereby, malignant tissue has to be fluid to a certain degree that cancer cells can move and divide [1]. Then again, a tumor mass might also contain solid regions that play a role in overall tumor stability and shape.

Tumor grading classifies tumors by tissue histology based on shape variation of cell nuclei and tissue morphology $[2,3]$. This classification is nowadays complemented by molecular markers [4], but it remains elusive how morphology and biochemistry relate to the biomechanics

\footnotetext{
*These authors contributed equally to this work.

†jkaes@uni-leipzig.de
}

Published by the American Physical Society under the terms of the Creative Commons Attribution 4.0 International license. Further distribution of this work must maintain attribution to the author(s) and the published article's title, journal citation, and DOI. of a tumor $[5,6]$. While genes and signaling pathways exert exquisite control over cell properties, mechanical interactions and the emerging collective behavior of cells play a critical role in sculpting collections of cells into functional three-dimensional (3D) tissues and organs [1,5,7-11], raising the question of how cells collectively shape the mechanical state of a tumor $[5,6]$.

Molecular cancer markers are mostly of prognostic and not predictive value [12], which means that the forecast of a patients fate relies on statistical data based on a large patient population but not on the individual state of the single patient. The ability of cancer cells to move in a specific tumor may be a predictive marker of a tumor's metastatic potential.

\section{TISSUE FLUIDITY AND CELL MOTION}

The hypothesis that cells can regulate bulk tissue fluidity is fueled by observations of 2D cell monolayers in cell culture environments. In these monolayers, jamming transitions straddle the line between solidlike and fluidlike tissue by changing cell shapes and number densities [13-20]. 
In fluidlike tissues, cells are able to move, comparable to particles in a fluid. In solidlike or jammed tissues, cells are locked in their positions, often acquiring compact shapes $[15,19]$. In classical jamming, a material solidifies into a disordered structure as a function of the packing fraction or temperature $[21,22]$. In cell monolayers, theory and experiments point to the existence of a novel shape-dependent transition [15,23].

Cells can also form nematic structures when they collectively align along their elongation axes [24]. Cell shapes may control cell proliferation [25]. Furthermore, they play -in conjunction with nucleus shape and stiffness $[26,27]$ - a central role in confined environments, where cells and nuclei deform and elongate to fit through narrow pores $[28,29]$.

The interest in the shape of cells in actual 3D tissues is also fueled from a medical perspective. In pathology, nuclear shape variations (also known as pleomorphism) are a fundamental grading criterion in cancer diagnosis [2], raising the question of clinical relevance of shapedependent cell and nucleus jamming theories.

Here, we find that mamma and cervix carcinoma consist of fluidlike and glassy, solidlike regions. We show that a marked slowdown of cell motility is reflected in solidlike bulk tissue mechanics. The first full account of 3D cell and nucleus shapes in tissues correlates tissue fluidity in solid tumors with static cell and nucleus shapes.

Previous studies in 3D systems focus on cell escape from multicellular tumor spheroids into the extracellular matrix (ECM) [30,31]. Another study in developmental biology finds a density-dependent motility transition in zebrafish embryos, where no cell shape dependency is reported [8]. These essential studies find transitions in single-cell motility correlated with local cell confinement but do not address the key question of how in solid tumors tissue pathological changes are reflected in tissue fluidity, cancer cell motility, and cell shape as well nucleus shape.

We concentrate on the ability of cells, in particular, cancer cells, to move in a dense microenvironment of surrounding cells and how this ability is modulated by bulk tissue fluidity. Since we focus on solid tumors that originate from epithelial tissue, cell clusters may be directly interconnected by cadherins or through ECM interspersed between the cells. Essentially, it does not make a difference to our results if cell-cell adhesion is mediated by cadherins or by collagen as a glue between the cells. We focus on compact cell clusters densely filled with cells and stained interstitial medium to estimate the space between cells. In the examined samples, we cannot detect significant visible extracellular space (see the Appendix). If one assumes interstitial space of a width $\frac{1}{2} \Delta$ around each cell of size $R_{C} \approx 8 \mu \mathrm{m}$, the volume fraction of this space is approximately $S_{C} \Delta /\left(2 * V_{C}\right) \approx 5 \%$, with $\Delta$ as the optical resolution $\Delta \approx 250 \mathrm{~nm}$ [for NA $=1.3$ as, e.g., in Fig. 2(a)]. Here, $S_{C}$ and $V_{C}$ are the surface and volume, respectively, of spheres of radius $R_{C}$. Optically nondetectable free space around cells, thus, cannot exceed approximately $5 \%$, which defines the parameter range for potential density effects. The volume fraction of cells is, thus, above both the random colloid jamming fraction of $\phi \approx 64 \%$ and close-packing fraction of $\phi \approx 74 \%[32,33]$.

We investigate the vital tissue of 16 resected tumors (12 cervix and four mamma carcinoma). Patients are informed and asked for their participation through an approved ethics votes (cervix ethics vote No. 090-10-19042010, mamma ethics vote No. 073-13-11032013). We find fluid as well as solid areas of cells in tumors of different staging, grading, and nodal status (see Table I in the Appendix). Further clinical trials are necessary to provide a meaningful correlation between fluidity and grading. Since a resected tumor is too large to be fully optically examined, we can only coarsely estimate the fraction of the different areas in a heterogeneous tumor. As a rough estimate, focusing on only cell-filled areas of the tumor, we find that $30 \%-40 \%$ of these cancer cell clusters behave as fluid using cell motility as an indicator of tissue fluidity. It is predicted that also cell proliferation can contribute to tissue fluidization $[34,35]$. Since a typical breast tumor has a tumor volume doubling time of about 150 days [36], we conclude that cell proliferation cannot contribute significantly to the observed effects, which occur in less than $12 \mathrm{~h}$. For the cell lines that we use, we measure that cell migration in the spheroids is a faster process than cell proliferation. Thus, tissue fluidization can be attributed to changes in motility and associated factors, such as contractility, cell-cell adhesion, and cell density. We confirm this conclusion by using mediuminhibiting cell proliferation.

We directly observe cell motility in primary human tumor explants from patients using a new cell-tracking technique, similar to a recent publication that investigates explanted mouse tumors [37]. Directly following surgery, tumors are cut into small pieces of typically around $50 \mu \mathrm{m}$ radius, and cell nuclei are labeled with the vital fluorescent stain SiR-DNA to record cell tracks.

The videos of tumor pieces show in the cancer cell clusters two distinctive types of areas, where collectively the cells either are immobile or can move within the $12 \mathrm{~h}$ observation time (videos are given as Supplemental Material [38]).

We record 4D-live stacks of the tumor explants and track cells in them during several hours of observation time. A representative example of this kind of observation is shown in Fig. 1.

In order to systematically investigate fluid-to-solid transitions in 3D tissues, we focus on cell spheroids, also called tumor spheroids, which are droplets of cells, which are filled to a volume fraction above classical colloid jamming. They are comparable in size to the primary tumor piece shown in Fig. 1 but are a reproducible system suited for quantitative investigation. They are built by letting cells 
(a)

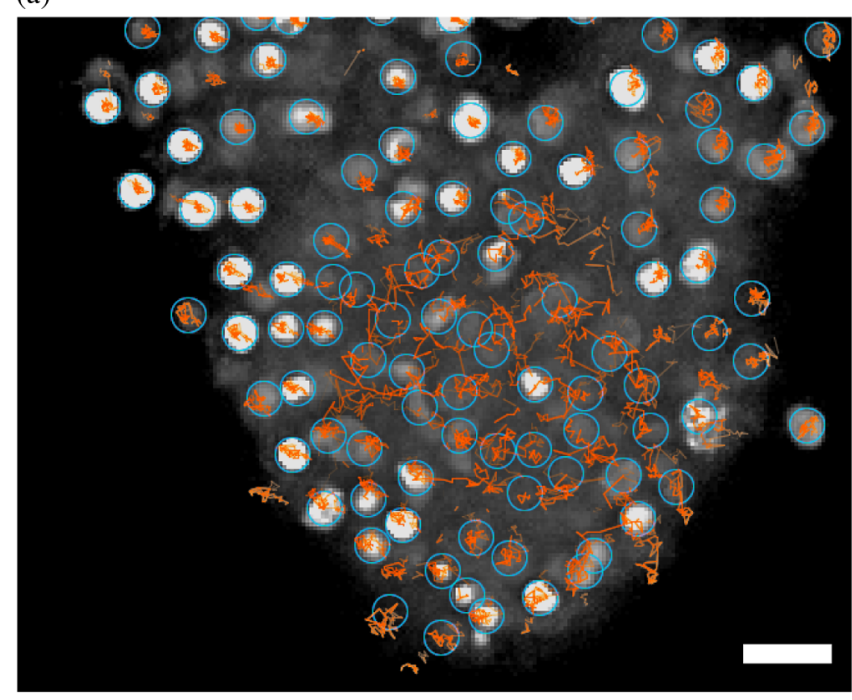

(b)

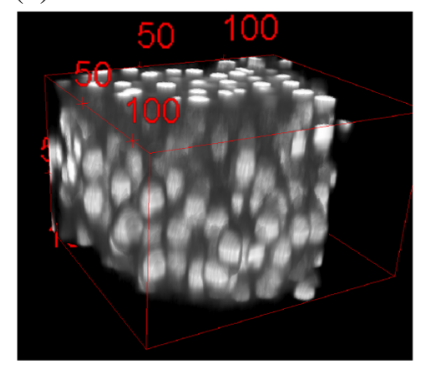

(c)

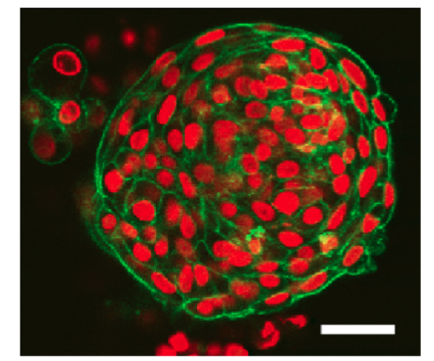

FIG. 1. Live cell tracking in primary human tumor explants shows regions of high and low cell motility. (a) Cell tracks in human, primary breast tumor tissue. Manually dissected pieces of a tumor are stained with a vital DNA stain and observed for $12 \mathrm{~h}$. While cells are very motile in some regions, others show stable cell arrangements, reminiscent of cell jamming. Tracking done using FIJI [39]. Scale bar, $20 \mu \mathrm{m}$. (b) The 3D view of the same tumor piece shows a compact, dense cluster of cancer cells. Scales in micrometers. (c) Confocal slice of a fixed and stained cervix tumor piece, which is optically cleared for a more detailed analysis. Actin stain (green) and DNA stain (red) reveal cell and nucleus shapes in a compact cell cluster. Cells can assume different shapes but are always densely packed. The extracellular space cannot exceed 5\%-6\%. Scale bar, $50 \mu \mathrm{m}$.

closely adhere to each other, forming a densely packed 3D system of active soft matter [Fig. 2(a)].

We study healthy epithelial MCF-10A and malignant MDA-MB-436 cell lines, both of which originate from breast glandular tissue. We have previously characterized their single-cell properties, finding that the malignant MDA-MB-436 cells are less adhesive in cell-cell contact experiments, express less E-cadherin, and are slightly softer than MCF-10A [40], which is typical of increased malignancy $[6,41]$.

The investigation of the tumor spheroids is organized as follows. We first characterize the 3D bulk mechanics during spheroid fusion, finding a fluid and a glassy, solidlike state.
In order to understand tissue fluidity on a microscopic level, we investigate single-cell motility in spheroids, and we quantify when and where they behave fluidlike or jammed. To link these global tissue states to cell properties, we develop the tools to fully image cell sizes and cell shapes in the 3D spheroids on a single-cell scale. We finally explain how the two spheroid types, the fluidlike and the solidlike, are both able to fuse together by employing two different modes of bulk tissue deformation.

\section{FLUID AND STALLED SPHEROID FUSION}

Collectively unjammed motile or jammed immobile cells are considered to cause fluid or solid tissues, respectively. Here, we present a fundamental experiment that permits us to relate freedom of local cellular motion with the fluid behavior of tissues. We characterize the dynamical bulk mechanical behavior of cell spheroids by droplet-fusion experiments. We place two spheroids of the same cell type and size next to each other and record their coalescence for 2 days.

For controlled experiments with defined homogenous cell populations, we use a breast cancer cell panel consisting of a mesenchymal, cancerous (MDA-MB-436) and an epithelial, healthy breast cell line (MCF-10A). Time series [Fig. 2(b)] and videos (Supplemental Material [38]) show that MCF-10A fusions continuously slow down and after about $12 \mathrm{~h}$ arrest in a state of partial coalescence. MDA-MB-436 fusions continue to progress as expected for the behavior of fluid droplets.

We quantify this process by the coordinate $\Theta$, defined as the angle between the line connecting the centers of the spherical caps and the line connecting the centers and the neck [Fig. 2(c), inset] [42]. As the fusion progresses, $\cos (\Theta)$ decays from 1 to 0 [Fig. 2(c)].

For passive Newtonian fluids, the fusion is driven by a surface tension $\sigma$ against a viscous drag $\eta$. A characteristic timescale $\tau_{\text {fusion }}=2 R_{S} \eta / \sigma$, given by $\sigma, \eta$, and spheroid radius $R_{S}$, governs the fusion progress [42]:

$$
\cos \Theta=\exp \left(-\frac{t}{\tau_{\text {fusion }}}\right) .
$$

The surface tension can arise from cell-cell adhesion but also from active cell contractility [43].

Although cell spheroids are surely not passive Newtonian fluids, this model accurately captures the fusion process of MDA-MB-436 spheroids [Fig. 2(c)], as well as the scaling $\tau_{\text {fusion }} \sim R_{S}$ of the fusion timescale with spheroid size (see the Appendix). The fusion timescale for the typical spheroids that we observe with radius $R_{S} \approx 100 \mu \mathrm{m}$ is about $\tau_{\text {fusion }}=20 \mathrm{~h}$.

In contrast, the arresting MCF-10A fusions are never described by a simple exponential decay and, instead, level off to a finite offset value after about $12 \mathrm{~h}$ of partial fusion [Fig. 2(c); see the Appendix]. 
(a)

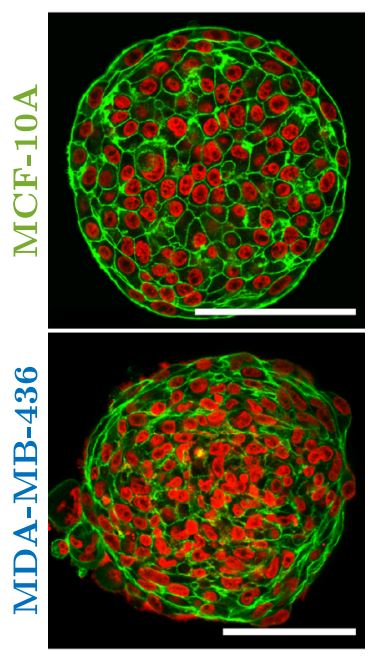

(b)

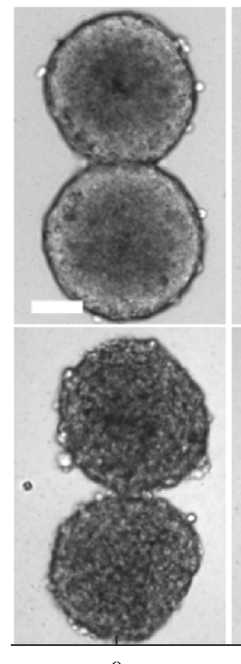

0

(c)

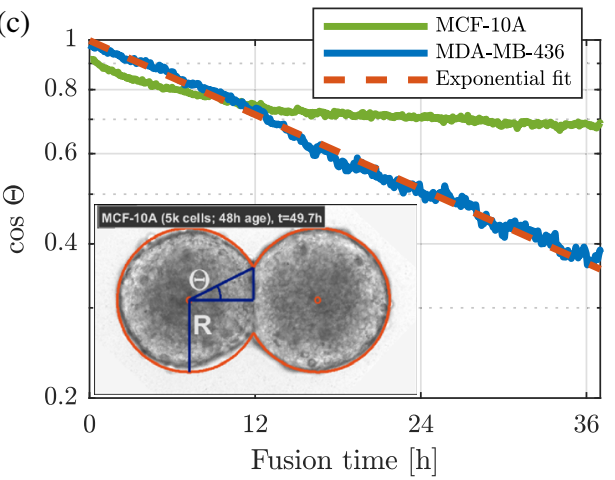

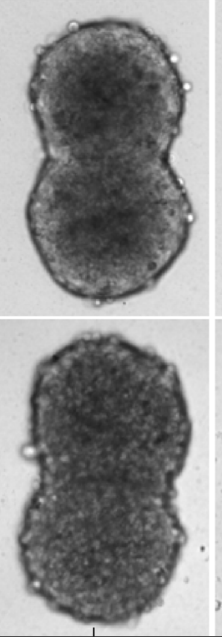

12

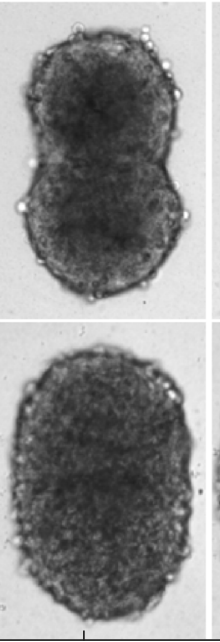

24

Fusion time [h]

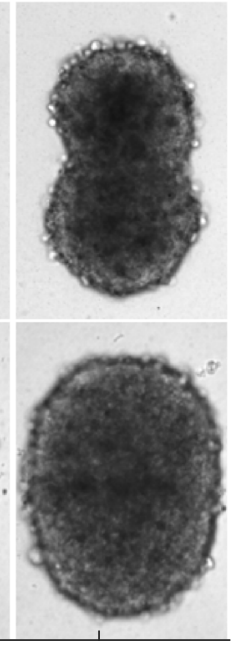

36

(d)

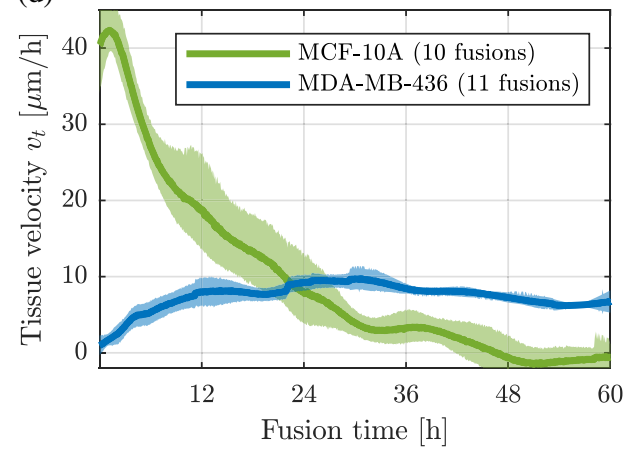

FIG. 2. Cell spheroids are an active multiparticle system close to $100 \%$ packing fraction. (a) Equatorial confocal sections of the two spheroid types used in this work: Epithelial-like MCF-10A and malignant MDA-MB-436 cells. Cell nuclei are marked in red. Actin, which assembles to a cortex beneath cell membranes, is marked in green. (Scale bars, $100 \mu \mathrm{m}$.) Global contrast is adjusted. (b) Time series of spheroid fusion. For MCF-10A cells, there is hardly any progress after $12 \mathrm{~h}$, while MDA-MB-436 spheroids fuse like fluid droplets. (Scale bar, $100 \mu \mathrm{m}$.) (c) Characterizing the fusion progress by $\Theta$, the angle between the fusion neck and the lines connecting the spheroid centers (see the inset). During fusion, $\cos (\Theta)$ runs from 1 (spheroids meet) to 0 (complete fusion). For MDA-MB-436, cos $(\Theta)$ follows an exponential decay as expected for a fluid [cf. Eq. (1)]. (d) Tissue flow velocities [Eq. (2)] during fusion experiments for the two tissue types show complete arrest (MCF-10A) and constant fluid flow (MDA-MB-436). Confidence intervals are from bootstrapping of the samples.

Rearranging Eq. (1) defines a quantity which we call the tissue flow velocity:

$$
v_{t}(t)=-2 R \frac{d}{d t} \ln \cos \Theta .
$$

For fluidlike fusion, $v_{t}$ is constant and reduces to the ratio of surface tension to viscosity, $v_{t}=\sigma \eta^{-1}$. Indeed, we observe that the cancerous, fluidlike MDA-MB-436 tissue velocities stay between 5 and $10 \mu \mathrm{mh}^{-1}$ during several days, indicating a permanent fluidization of the tissues on the typical fusion timescales of hours to days.

MCF-10A fusions start off with distinctly higher velocities, followed by a critical glasslike slowdown of the fusion dynamics [Fig. 2(d)], where the bulk tissue flow dynamics arrests. The strength of this deviation from fluid behavior depends on spheroid size and age (see the Appendix).
We cannot extract a characteristic timescale from the fusion experiments in terms of passive, Newtonian fluidity as in Eq. (1).

The complex functional form of the epithelial MCF-10A fusion data can be rationalized in several ways. It could be that the MCF-10A cells change their properties from solidlike to fluidlike as a function of the distance from the center of the aggregate; i.e., there is a hard core and a fluid shell. Alternatively, the behavior could arise from the interactions between two homogeneous but soft solids, which partially fuse due to large effective surface energies caused by active cell contractility, e.g., by overcoming a yield stress near the contact neck of the two spheroids.

The generation of ECM proteins by the cells themselves could possibly contribute to the solidification of the 
spheroids, for instance, by sticking cells together. However, we do not find with several approaches, including atomic force microscopy, detectable amounts of collagen within spheroids (see the Appendix), and neither do other groups [30].

As another option, the cells could change their properties as a function of time, which would be reflected by the bulk fusion dynamics. For instance, the tissue surface tension that drives the spheroid fusion could vanish over time. The strongly elongated cells on the surface of MCF-10A spheroids [Fig. 2(a)] indicate such surface tensions. These are still present after 2 days of culture, when the fusion speed has already dropped significantly. Moreover, spheroids never become unstable, even if exposed to external mechanical stress, e.g., by pipetting. These observations rule out the explanation that surface tensions would drop to zero. It is also disfavored by observations that the fusions of one-dayold spheroids arrest after $12 \mathrm{~h}$, while fusions of two-day-old spheroids still begin, although the first fusions had already arrested. A homogeneous viscosity increase with time could also not explain these observations.

Altogether, this result suggests that partially solid behavior in MCF-10A spheroids inhibits complete fusion. The fusion data suggest that, on the level of bulk mechanics, the MDA-MB-436 spheroids have predominantly fluid properties. As the next step, we connect the solid and fluid behavior to cell motility.

\section{LIVE OBSERVATION OF FLUID AND IMMOTILE CELLS}

We then investigate single-cell motility in the 3D cell clusters to test whether cells are free to move as particles like in a fluid or whether they have stable positions with respect to each other as in a solid.

Similar to the investigated primary tumor samples, we do not find large amounts of free volume between cells in either spheroid type. The actin cortex, shown in Fig. 2(a), assembles directly beneath cell membranes and is around $200 \mathrm{~nm}$ thick [44]. Limited by the optical resolution $d_{x} \approx$ $0.6 \lambda / \mathrm{NA} \approx 250 \mathrm{~nm}$ [for $\mathrm{NA}=1.3$ as in Fig. 2(a)], we typically cannot resolve two separate actin cortices of neighboring cells in the spheroids. Furthermore, the cell lines that we use adhere to each other using E-, N-, and Pcadherins [40]. The extracellular part of these junctions has a typical width of $25 \mathrm{~nm}$ [45]. For intercellular gaps of either 25 or $250 \mathrm{~nm}$ width, the volume fraction occupied by intercellular space is about $0.5 \%$ or $5 \%$, respectively. This estimate of extracellular space underlines that the cells occupy a volume fraction well above classical hard sphere jamming at volume fraction $\Phi_{c} \approx 0.64$ [46].

The limited extracellular space agrees with our finding that no detectable ECM is present. We cannot claim that cell density is precisely at volume fraction one. Nevertheless, a cell must deform to be able to move in such a dense microenvironment.
We thus employ the same technique for cell tracking as for the observation of cancer cells in primary tumor explants (Fig. 1). We record 4D-live stacks of spheroids of both cell types [Fig. 3(a)] for $6 \mathrm{~h}$. To be able to record data throughout the whole spheroids, we observe spheroids of size $R_{S} \approx 50 \mu \mathrm{m}$. Note that, for a given cell line, we see the same fluid or glassy fusion dynamics for spheroids of different sizes (Supplemental Fig. 7).

Video microscopy shows that cells move freely and randomly through MDA-MB-436 spheroids, despite the dense packing (Fig. 3; see videos given as Supplemental Material [38]). Figure 3(b) closely follows the track of the nucleus of a motile cell. The nucleus is a cell's most rigid structure. The cell nucleus has to squeeze so that the cancer cell can move through the dense microenvironment of surrounding cells. The nucleus undergoes visible deformations from frame to frame. It travels a distance of $d \approx 40 \mu \mathrm{m}$ in $2 \mathrm{~h}$, with a speed even comparable to cells moving freely on tissue culture substrates. This microscopy shows that (i) cancer cells can move through a densely packed 3D aggregate and (ii) this motion is accompanied by nucleus deformation and, as nuclei occupy a large part of the cell volume, by deformation of the cells.

During squeezing events, cell nuclei become highly deformed and get very close to each other [Figs. 3(a) and 3(b)], which obscures automatic cell tracking. As a consequence, we manually track 8-10 cells per spheroid [Figs. 3(c) and 3(d)]. In the fluid MDA-MB-436 spheroids, half of the cells displace by more than one cell radius after about $4 \mathrm{~h}$, showing that they are not caged on this timescale. During the $6 \mathrm{~h}$ of observation, some cells consequently leave the focal plane, so we manually follow them through different planes.

Cell motion is clearly enhanced in the aggregates of the breast cancer cell line with respect to the healthy epithelial cell line. How does this motion relate to the bulk behavior determined by spheroid fusion? The Lindemann criterion [47] discerns between solid and fluid states based on local, single-particle motion. It states that a material fluidizes when particle motion exceeds a threshold fraction $\delta_{L}$ of the interparticle distance. The value $\delta_{L}$ typically lies in the range from $0.05<\delta_{L}<0.22$ for atomic and molecular systems [47-50].

In the fluid spheroids, cells typically have aspect ratios (ARs) in the range of 1.5-2, as we show in the next section and the Appendix (Figs. 4 and 13). Their longest half-axis is, thus, typically elongated by a factor of about 2 , limiting the maximal distance for the freedom of a nucleus to move. Taking this elongation into consideration, we assume one (shape-independent) equivalent cell radius $R_{C}$ [defined below in Eq. (4)] as a useful estimate for the Lindemann threshold $\delta_{L}$ for nucleus movement in a dense 3D cell cluster, since this assumption permits fluid exchange of positions.

In the MDA-MB-436 spheroids, displacements clearly exceed the threshold of one equivalent cell radius $R_{C}$ within $6 \mathrm{~h}$ [Fig. 3(d)] and fulfill our Lindemann fluidity criterion in 


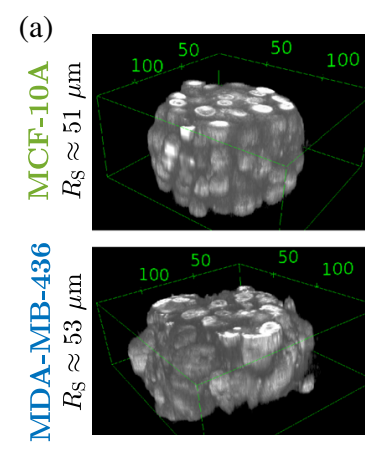

(c)
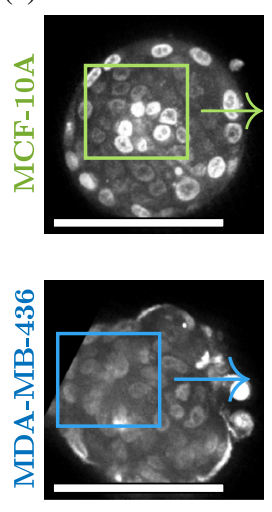

(b)
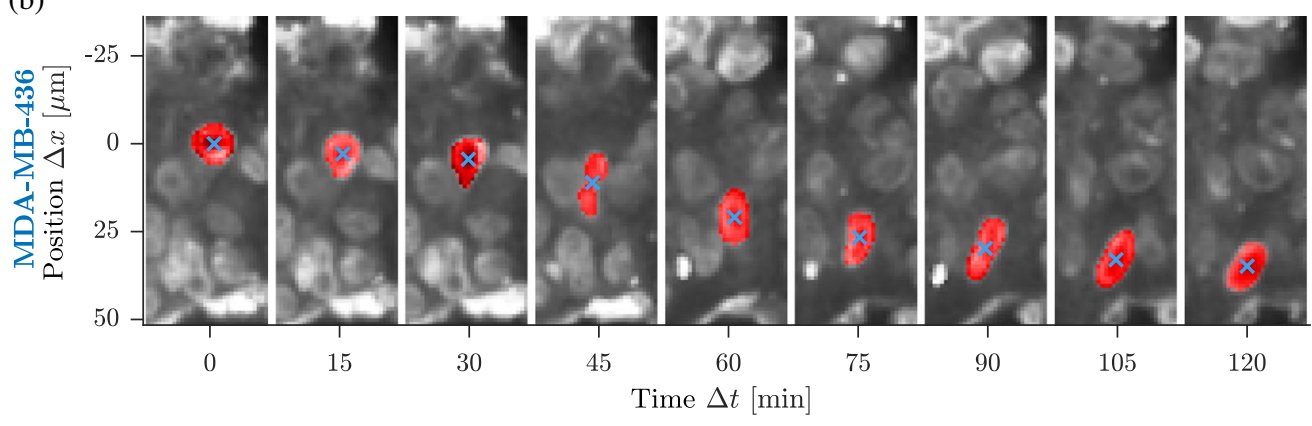

(d)
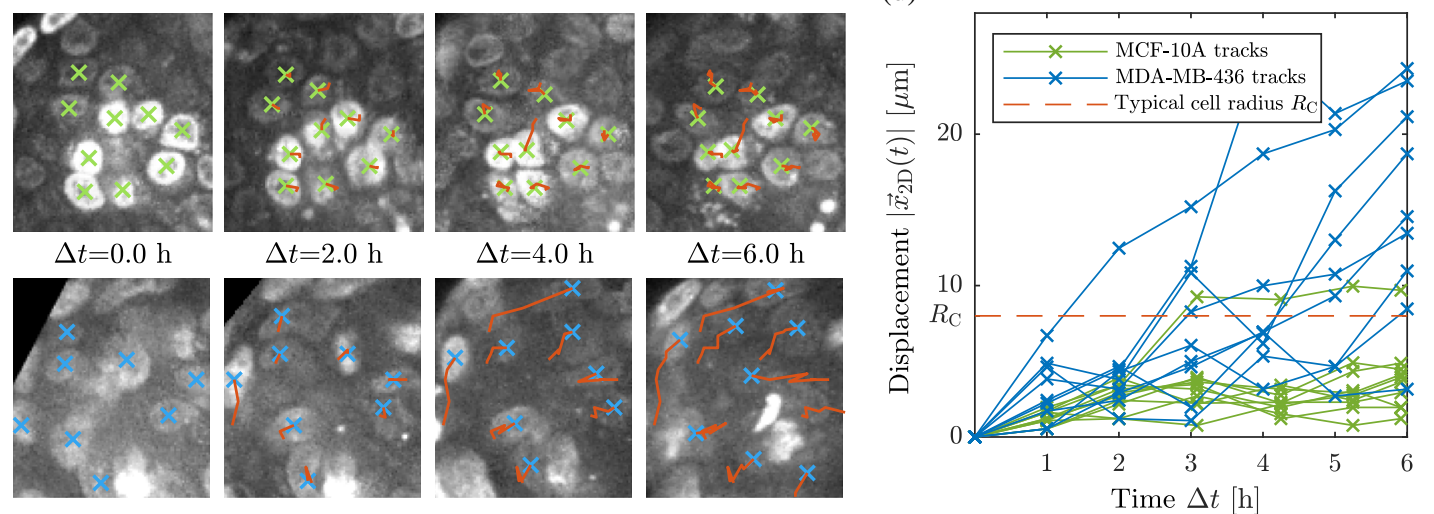

FIG. 3. Live observation of spheroids shows fluid and jammed cells. (a) Confocal 3D snapshots of spheroids $(n \approx 200$ cells). For $4 \mathrm{D}$ time stacks, $z$ sections of total height $\Delta z \approx 60 \mu \mathrm{m}$ are recorded every 5 min during several hours. Cell nuclei are stained with SiR-DNA. $3 \mathrm{D}$ renderings are created with FIJI [39] plugin 3D viewer. (b) Time series of the motion of a cancer cell's nucleus in a densely packed spheroid. For better visibility, the cell of interest is marked in red. Images come from a total of seven different $z$ slices that the nucleus travels through over the course of $120 \mathrm{~min}$. ( $\Delta z \approx 24 \mu \mathrm{m}$.) The total distance that the nucleus travels is $\gtrsim 40 \mu \mathrm{m}$. (c) Manual cell tracking results in spheroids of both types, in the marked square areas, for 8-10 cells each. The nuclei of the healthy MCF-10A cells stay at their position in the spheroid, while the nuclei of malignant MDA-MB-436 cancer cells move and leave their positions. Scale bars, $100 \mu \mathrm{m}$. Global contrast is adjusted. (d) In-plane displacements of the tracks shown in (c).

agreement with our finding that the bulk of the spheroid behaves as fluid. In MCF-10A spheroids, this criterion is not fulfilled, in accord with the solid behavior of the core of the aggregates. Note that, while the threshold value $R_{C}$ is an estimate, our assessment of fluidlike MDA-MB-436 and solidlike MCF-10A does not change if we choose a value somewhat lower or higher than $R_{C}$ as the threshold [Fig. 3(d)].

The average time cells need to move by one cell radius is an estimate for the fluidization timescale $\tau_{\text {fluid }} \approx 4 \mathrm{~h}$ of the tissue. Using a more sophisticated autocorrelation approach involving data from many spheroids, we verify that this timescale estimate is correct (see the Appendix).

Cells in the MCF-10A spheroids are drastically slowed down and drop in their motile range below the Lindemann criterion. Most of the cells stay very close to their positions and do not leave the focal plane [Fig. 3(c)]. Only one out of ten manually tracked cells moves by more than one cell radius during $6 \mathrm{~h}$ of observation [Fig. 3(d)].

At the end of our tracking experiments, we use the wetting behavior of the spheroids on substrates as a viability assay, showing that cell migration is restored and that our spheroids have no necrotic core. Thus, loss of viability can be ruled out for cell arrest. The cells are transiently immobilized by their dense environment.

MCF-10A cells are in no way inherently immobile per se and do not lack the molecular machinery to move. On 2D tissue culture substrates, they reach average speeds of more than $30 \mu \mathrm{m} \mathrm{h}^{-1}$. Furthermore, the outer cell layers of MCF-10A spheroids, which feel less the constraints of dense cell packing, initially fuse much quicker than MDA-MB-436 spheroids [Fig. 2(d)]. Compared to free motion, epithelial cells in the dense microenvironment spheroids are slowed down by more than one order of magnitude, moving at an average speed of about $1 \mu \mathrm{mh}^{-1}$ [Fig. 3(d)].

As previously expected, the bulk fluidity in 3D cell aggregates as measured in the fusion experiments relates to the ability of cells to move freely. Like in a glass, the drastic slowing down of cell motion leads to a solid behavior.

We now investigate how the glassy and the fluid states relate to cell-jamming concepts, before we take a closer look at the fusion process and, more specifically, how the nearly immotile MCF-10A cells can form partially fusing spheroids. 
(a)

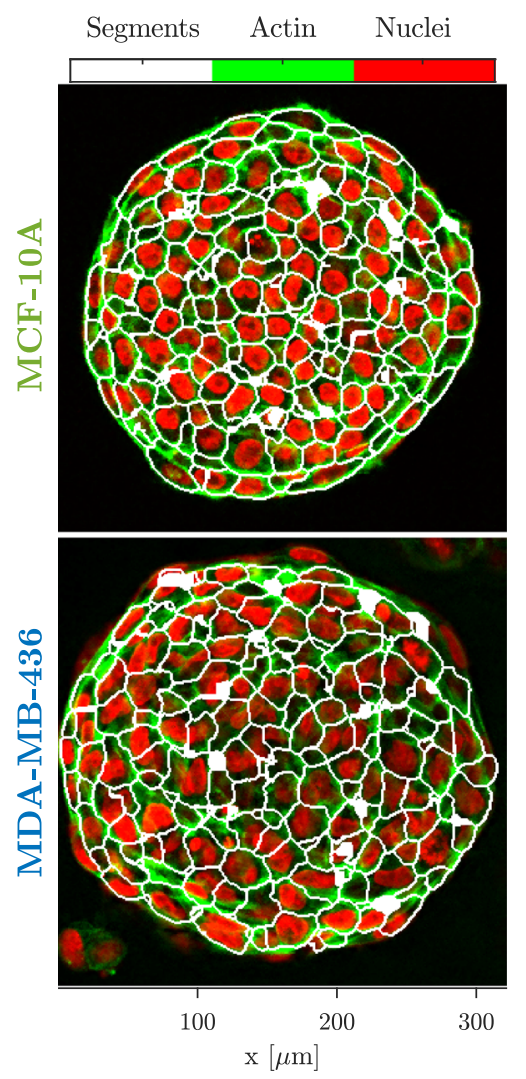

(b) $\quad 3 \mathrm{D}$ cell shape index $s_{\mathrm{E}}$ (neighbourhood median)
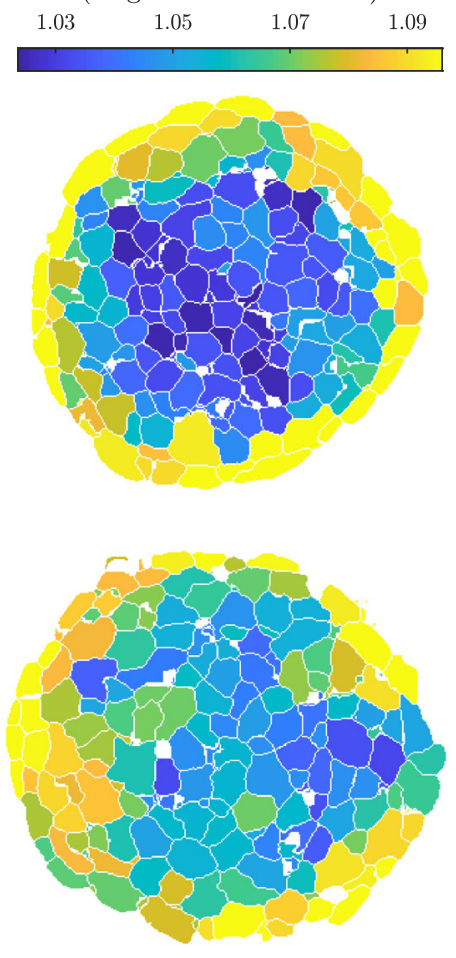

(c)
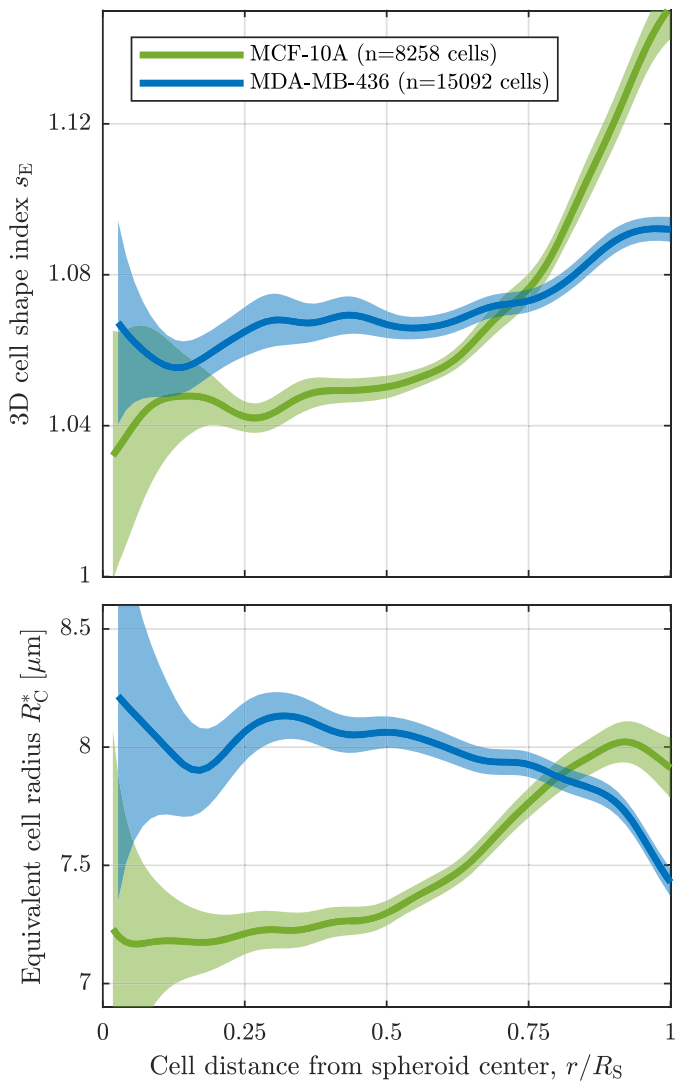

FIG. 4. 3D segmentation reveals cell shapes and sizes. (a) Overlay of the 3D segmentations on the fluorescence pictures. The white spots appear when a segment boundary is coplanar with the image section and, thus, do not represent holes in the aggregates. Global contrast is adjusted. (b) Heat maps of 3D shape indices $s_{E}$ in the spheroids (shown for every cell is the median over all neighbor cells). (c) Radial distribution of 3D cell shape indices $s_{E}$ [Eq. (3)] and equivalent cell radius $R_{C}^{*}$ [Eq. (4)] in the spheroids, averaged for 3-4 spheroids of either cell type. Shaded areas are confidence intervals of moving bins of width $0.2 R_{S}(\mathrm{FWHM})$ for $p=0.05$.

\section{CELL SHAPES AND SIZES IN THE AGGREGATES}

The data by Mongera et al. clearly show that densityinduced cell jamming is possible in a developing embryo [8]. The situation during the malignant transformation of a healthy adult tissue is different, since the cell density is close to volume fraction one with a small variable range of $5 \%$. It has been suggested that tumor progression involves an unjamming transition, however, raising the question of how cell motility is possible in such a crowded environment. Pathologic changes of the nucleus in size and shape are characteristic to cancer [2]. Cancer cell motility in vitro highly depends on the shape and stiffness of their nuclei [26,27]. Experimental evidence in substrate-based cell monolayers and models link 2D cell jamming to cell shapes $[1,15,19,23,51]$. Some cell-jamming studies invoke cell number densities as the driving factor [13,20]. A recent 3D-Voronoi model suggests that cell shape arguments can be generalized to physiologic 3D situations [52]. These findings demand that we investigate how cell density and cell as well as nucleus shape change in cancer cell unjamming.
We quantify how cell shapes and number densities correlate to the different motility states that we find. To investigate these quantities with a high level of precision, we fix, stain, and optically clear spheroids of both spheroid types. Equatorial slices of full 3D stacks are shown in Figs. 2(a) and 4(a). Actin and nuclear stains reveal the cell and nucleus shapes within the spheroids. Already by visual inspection, cells in the fluid MDA-MB-436 spheroids appear on average more elongated than in the MCF-10A spheroids, consistent with our observation of cells with elongated nuclei moving through the fluid tissue [Fig. 3(a)].

We use the actin and nuclei signals as input for a selfdeveloped high-precision segmentation algorithm, which digitally decomposes the 3D image stack into single-cell volume segments [Fig. 4(a)]. The segmentation algorithm is described in detail in the Appendix. Using the pointspread function of our confocal microscope, realistic noise, and the impact of refractive index mismatches [53] to blur simulated pictures of cell spheroids, we verify the accuracy of the segmentation algorithm (see the Appendix).

We quantify all cell shapes in every image stack by the (size-independent) $3 \mathrm{D}$ shape index $s_{E}$, which is a dimensionless surface-to-volume ratio: 


$$
s_{E}=(36 \pi)^{-1 / 3} S_{E} V_{E}^{-2 / 3} .
$$

Here, $S_{E}$ and $V_{E}$ are the surface area and volume, respectively, of the corresponding ellipsoid that has the same second volume moment as the cell segments. The second volume moment is closely related to the inertia tensor and robustly grasps the overall shape, while it is insensitive to surface roughness. The shape index $s_{E}$ is normalized such that, for spherical cells, $s_{E}=1$, and for, e.g., a typical chicken egg, $s_{E} \approx 1.02$ [54]. A 3D-Voronoi model has recently predicted a jamming threshold at $s_{E}^{*} \approx$ 1.003-1.013 (Ref. [52]; cf. the Appendix), meaning that cell motility would completely stop below this value.

Cell shapes can also be quantified by other common measures, such as aspect ratios [19], Voronoi tessellation shapes [23,52], or the shape index of the actual cell surface and volume as detected by the algorithm. We compare all these cell shape measures in the Appendix. Although precise numerical values rely on which measure is used, a set of observations independent of the specific measure emerges that leads to the same conclusions. The further discussion in this manuscript thus concentrates on the ellipsoid cell shape index $s_{E}$.

We investigate the influence of imaging noise, optical resolution, and refractive index mismatches [53] on our results, finding that they are surprisingly stable and that the values are no artifacts of our imaging methods. We verify our segmentation on synthetic data. Details on these investigations are presented in the Appendix.

We further quantify cell sizes by the (shape-independent) equivalent cell radius $R_{C}^{*}$, given by the cell segment volume $V_{C}$ :

$$
R_{C}^{*}=\left(\frac{3}{4 \pi} V_{C}\right)^{1 / 3} \text {. }
$$

\section{CELLS SHAPES REFLECT AGGREGATE FLUIDITY}

Cancer cells within the fluidlike MDA-MB-436 spheroids, which are motile, are on average more elongated than immobile epithelial cells in the solidlike core of MCF-10A spheroids [Fig. 4(c)]. We find a cell shape index $s_{E}^{436}=$ $1.067 \pm 0.002$ (mean $\pm S E, n=2254$ ) for cells within the spheroid core (cells with $r<0.5 R_{S}$, where $R_{S}$ is the spheroid radius).

The immobile cells in the jammed MCF-10A spheroid are significantly less elongated with $s_{E}^{10 A}=1.048 \pm 0.001$ for the core cells $(n=1556)$.

In all cases, we observe average cell shapes [Fig. 4(c)] above the proposed threshold value for 3D cell jamming [52]. Nevertheless, some cell clusters in the jammed samples come very close [Fig. 4(b)]. We also do not see total jamming, i.e., full arrest of cell motion. The drastic slowing down of cell motion is sufficient that the core of MCF-10A spheroids behaves like a glasslike solid in the fusion experiments. The degree of bulk tissue fluidity relates to cell motility, which correlates with elongated cell shape.
We further see that both tissues have more elongated cells toward the edges of the aggregates. This elongation is the signature of boundary effects, such as tensions holding the cells back to form a stable boundary.

\section{CELLS IN THE JAMMED SAMPLE ARE PACKED AT A HIGHER NUMBER DENSITY}

As already mentioned, density-induced cell jamming in 3D has been already observed. Cell number densities are proposed to drive cell jamming $[13,20]$. In the spheroids that we study, cells are practically volume filling; however, changes in cell size modify the cell number density per volume.

We find smaller cells-and, thus, higher cell number densities - in the core of the jammed MCF-10A spheroids [Fig. 4(b)], when compared to the unjammed MDA-MB436 spheroids. For MCF-10A cells in the core $\left(r<0.5 R_{S}\right)$, we find $R_{C}^{*}=7.2 \pm 0.8 \mu \mathrm{m}(\mathrm{mean} \pm \mathrm{SD})$, and for MDAMB-436 $R_{C}^{*}=8.1 \pm 1.3 \mu \mathrm{m}$. To calculate these values, we neglect intercellular space.

This result raises the question if MCF-10A cells are smaller per se or just more densely packed in the spheroid core. To answer this question, we compare these values to measurements of suspended, single cells. We find that single, suspended MCF-10A cells are bigger than the cells in the core of the spheroids $\left(R_{C} \approx 8.7 \pm 1.0\right)$. They are also bigger than MDA-MB-436 cells $\left(R_{C} \approx 8.1 \pm 1.2\right)$ (see Supplemental Material of Ref. [40]). This result indicates that MCF-10A cells in the core are indeed more densely packed or compressed to a higher number density. The cell sizes of the cancerous MDA-MB-436 cells within the spheroids are, by contrast, indistinguishable from the value we find for single, suspended cells ( 8.3 vs $8.1 \mu \mathrm{m})$.

This difference in cell size of the MCF-10A cells $R_{C}^{*}$ is small when compared to the differences in $2 \mathrm{D}$ monolayer jamming [13]. Differences in $R_{C}^{*}$, however, translate into larger differences in cell number densities $n / V \sim R_{C}^{*-3}$. The increased cell packing in MCF-10A spheroids could, thus, contribute to the reduced cell motility.

Reduced cell sizes within aggregates are linked to fluid transfer through gap junctions between cells [31]. It could also be linked to epithelial contractile behavior [55]. This result may be enhanced by an effect we observe during spheroid formation. MCF-10A cells form a 3D contractile actin rim around the spheroid, which may condense the cells (Fig. 5).

The complex mechanical interplay between softer outer cytoplasm and rigid inner nucleus may have a decisive impact on cell jamming. The weaker cytoplasm may substitute through its deformability for free volume, which could explain cell unjamming at the observed high cellular volume fractions. The nuclei of MDA-MB-436 cells appear larger than normal MCF-10A cells, which is typical for cancer progression [56]. In the densely packed 3D spheroids of nearly volume filling cells, the higher 


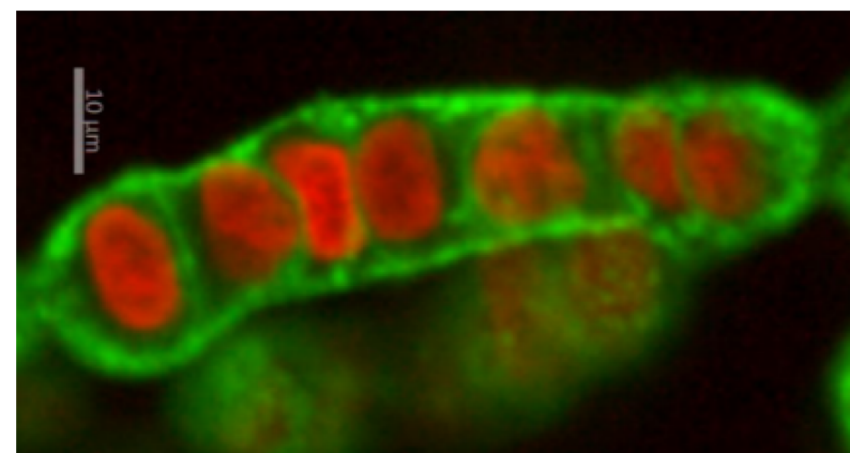

FIG. 5. During the generation of MCF-10A clusters, the formation of a pronounced collective actin cortex becomes visible. Actin marked in green, nuclei in red. This observation does not happen in MDA-MB-436 spheroids.

number density in the jammed core of MCF-10A aggregates with respect to the fluid MDA-MB-436 clusters may be caused by the smaller, probably more rigid, nuclei.

\section{CELL SHAPES IN FUSING SPHEROIDS}

We find that, although the two tissues are in different states, a fluid and a glassy, solidlike state (Figs. 1 and 4), pairs of spheroids at least partially fuse together (Fig. 2). How do the two different states accomplish the bulk deformation necessary for (partial) fusion?

The malignant MDA-MB-436 spheroids are fluidized by motile cells (Fig. 3). The fluidization timescale $\tau_{\text {fluid }} \approx 4 \mathrm{~h}$ is much faster than the spheroid fusion timescale $\tau_{\text {fusion }} \approx 30 \mathrm{~h}$ [Eq. (1) and Fig. 2(c)]. The stress relaxation within the spheroids is, thus, faster than the bulk deformation rate during fusion. From these results, we conclude that in the unjammed tissue deforming stress can dissipate like in a fluid during fusion.

The situation is far more complex in MCF-10A spheroids with jammed or at least drastically slowed down cells [Figs. 3(c) and 3(d)]. MCF-10A fusion, however, initially progresses even faster than in MDA-MB-436 [Figs. 2(c) (a)

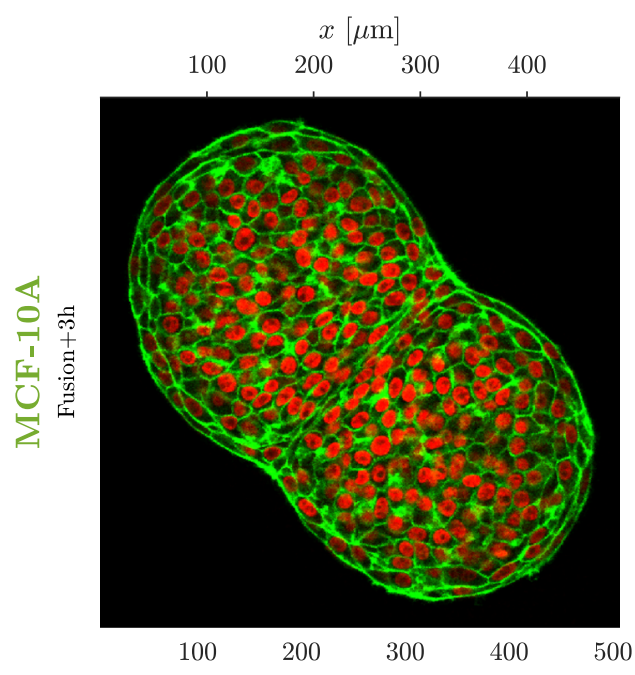

(b)

3D-shape index $s_{\mathrm{E}}$ (neighbourhood median)
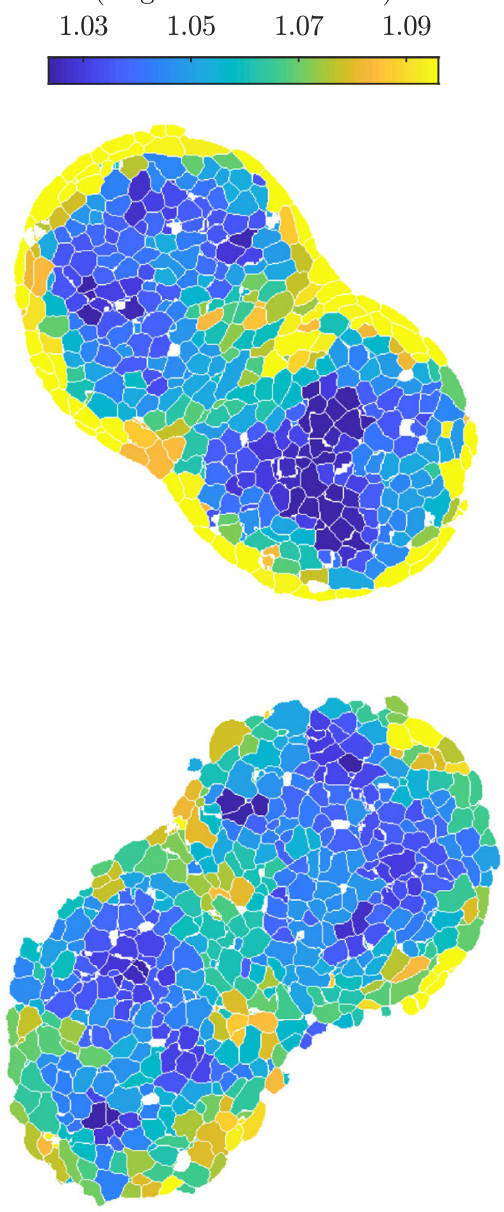

(c) Nematic order $S_{\text {short }}$ (neighbourhood median) $\begin{array}{lllll}0 & 0.2 & 0.4 & 0.6 & 0.8\end{array}$
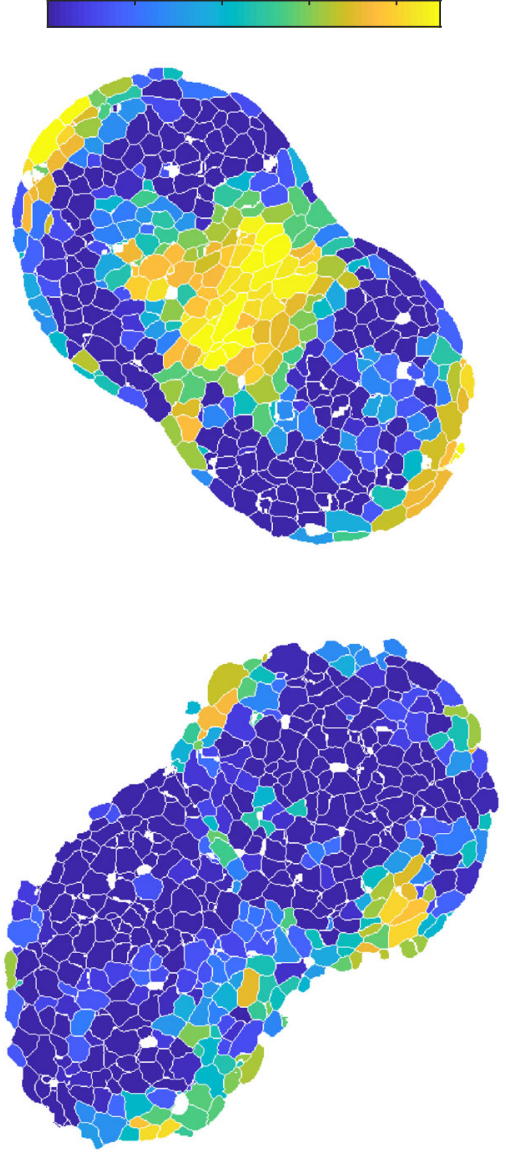

FIG. 6. Cell shapes during spheroid fusion show collective cell alignments and disordered behavior. (a) Equatorial confocal sections of spheroids fixed during fusion. Staining as in Fig. 4 (actin marked in green, nuclei in red). Global contrast is adjusted. (b) Cell shape index $s_{E}$ [Eq. (3)] during fusion [as in Fig. 4(b)]. (c) Nematic order parameter $S$ [Eq. (5)] for the cellular short axes (shown for every cell is the median over all neighbor cells). 
and 2(d)], seemingly at odds with our expectation of a solidlike tissue behavior. This result raises the question if and how MCF-10A spheroids specifically adapt to allow for the fast initial cell displacements during fusion.

To examine the spheroid architecture during fusion, we apply the segmentation routine to fusing spheroids of both cell types at comparable stages of fusion. We observe that, in MCF-10A spheroids, cells along the contact area between the two fusing spheroids become collectively aligned [Fig. 6(a)]. We quantify this collective alignment using the nematic order parameter $S$ [Fig. 6(c)]:

$$
S=\frac{1}{2}\left(3 \cos ^{2} \alpha-1\right) .
$$

Here, $\alpha$ is the angle between the cell main axis and the local director, which is taken as the average main axis of all neighboring cells. As each cell has three main axes, $S$ can be calculated for short, middle, or long axis alignment.

We find a strong collective short axis alignment $S_{\text {short }}$ in fusing MCF-10A spheroids [Fig. 6(c)]. Near the fusion neck, short axes are collectively oriented, pointing toward the fusion neck. Cells get flat, oblate shapes. The other two axes are largely unaligned (data not shown).

We see that the two tissues employ different strategies to achieve the material transport during the initial steps of spheroid fusion. In both types, cells along the fusion boundary are elongated [Fig. 6(b)]. The difference is that, in the solidlike epithelial sample, the fusion elicits an intermediate state of collective deformation, leaving a characteristic footprint of elongated and aligned cells at the fusion neck. This deformation can point toward an elevated cell motility, i.e., a fluidization process, or to a collective solid deformation of the soft cell collective. In the fluid MDA-MB436 samples, the boundary between the two spheroids is much less defined. The nematiclike cell alignment is absent in these spheroids; they are "fluid enough" to fuse.

Recent findings on cell contractility from our and other groups [55,57] may explain this different fusion behavior. We newly find that epithelial cells such as MCF-10A cells can contract in a substrate-independent manner based on the actin cortex. In cell clusters, epithelial cells form a contractile collective actomyosin rim around the spheroid, which contributes to the tissue surface tension [43]. This behavior becomes most obvious during spheroid formation (Fig. 5). Meanwhile, cancer cells like MDA-MB-436 cells exhibit cortical contractility and do not form a collective contractile rim. This difference explains why initial fusion for MCF-10A spheroids progresses faster. Fusion stalls when the contractile forces are balanced by the elastic stress from the deformation, since the bulk behaves like an elastic solid.

\section{RELATION BETWEEN CELL AND NUCLEUS SHAPE}

In single-cell motion through narrow constrictions, nucleus jamming is the ultimate constraint, since the
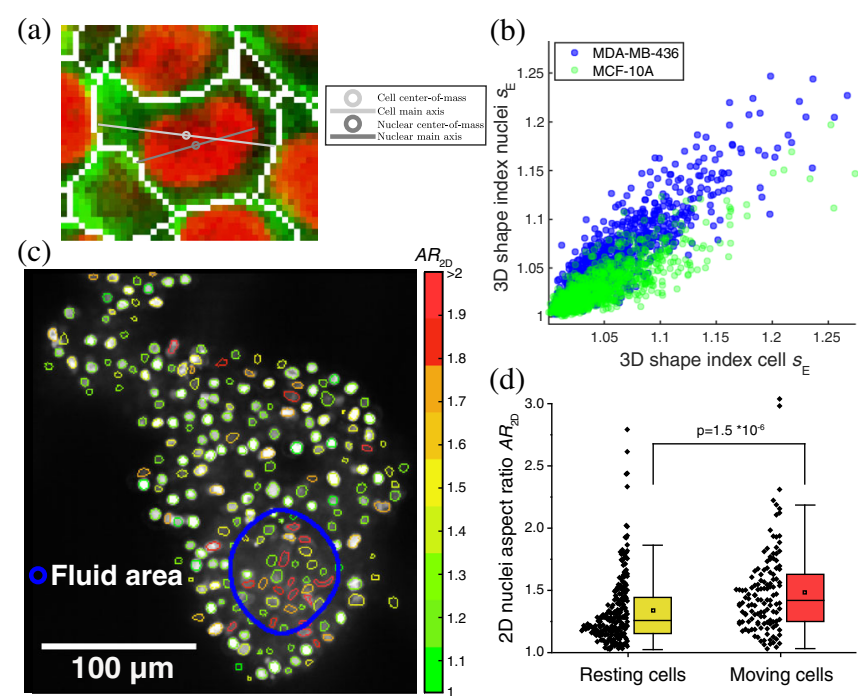

FIG. 7. The close relation between cell shapes and nuclear shapes in cell clusters links cell unjamming to cancer grading. (a) Close-up of a confocal section of an MCF-10A spheroid. Green, actin stain; red, DNA stain; white, 3D-segmentation boundaries. The main long axes shown for the cell and nucleus are projections onto the image plane. (b) In both cell types, 3D shape indices of cells $s_{E}^{\text {cell }}$ and 3D shape indices of nuclei $s_{E}^{\text {nuc }}$ are highly correlated. (c) In primary samples, fluid areas (blue region; assessed by visual inspection) show more elongated nuclei. (d) Significantly higher degree of nuclear shape elongation in fluid areas. The shape and appearance of the nucleus judged by the pathologist are part of the standard procedure in the evaluation of histological slides.

nucleus is more rigid than the surrounding cytoplasm $[26,27,56,58]$. Malignant cell nuclei are larger and change their molecular architecture to become softer [56], and their ability to move in dense environments depends on the nucleus shape. As illustrated in Fig. 3(b), nucleus shape seems to play a central role in cell motility in dense cell clusters.

Consequentially, we detect [see Fig. 7(a)] the 3D shape index of the nuclei $s_{E}^{\text {nuc }}$ in the spheroids and compare them to the shape index of cells $s_{E}^{\text {cell }}$. Both shape indices strongly correlate with each and linearly depend on each other [see Fig. 7(b)]. In the fluid MDA-MB-436 cells, the nucleus 3D shape increases stronger with increasing 3D cell shape index with respect to the jammed MCF-10A cells, which may be caused by MDA-MB-436's larger, more dominant nuclei. In agreement with the central role for cancer cell migration in the ECM, the nucleus shape plays also a key role for migration in cell clusters. Moreover, elongated cell shape and nucleus shape are a reliable indicator for tissues where increasing cell motility causes a more fluid state. In primary breast and cervix tumor samples, areas with motile cancer cells with respect to the jammed areas contain cells with more elongated nuclei [Figs. 7(c) and 7(d)]. Cancer cell nuclei are larger and softer [56]. Thus, it is to be 
expected that these nuclei have to deform more when cancer cells move through the dense matrix of surrounding cells. In all solid tumor stages that we investigate, we find solid and fluid tissue. The jammed aggregates can form a solid backbone, and the fluid regions permit proliferation and motility.

\section{TISSUE FLUIDITY IN CARCINOMA}

Real tumors are highly heterogeneous with respect to cell genetics and phenotypes [59]. Besides cells and ECM, we find that cell aggregates in tumors are also mechanically heterogeneous (Fig. 1). We identify two distinct states of tissue fluidity in breast as well as cervix carcinoma with the help of 3D tumor spheroids as the model system. In analogy to the solid behavior in benign epithelial spheroids, we find tumor regions in an amorphous solidlike state with suppressed cell motility contributing to a stable, palpable tumor backbone. This condition is characterized by drastically reduced single-cell motility and bulk tissue velocity due to a glassy slowdown. The other part of cell aggregates in tumors shows fluid properties as a prerequisite for high single-cell motility and proliferation in similarity to tumor spheroids of unjammed cancer cell lines. Thus, tissue fluidity is already necessary at early stages of tumor progression.

Mongera et al. [8] find in embryonal development a celljamming transition facilitated by an increase in cell density, since the ability of tissues to behave like a solid is essential to form stable organs and structures. Tumor progression, however, requires cell unjamming in a very dense environment with practically no intercellular space for cells to move and divide. In our cell aggregates, this space is less than 5\%. The tumor simultaneously requires a solid scaffold for stability against its microenvironment. Strongly suppressed cancer cell motility is thereby sufficient for solidlike behavior, but a complete arrest is not necessary.

Conventional jamming is induced by increasing density. In the cancer cell clusters in tumors, the cell density is clearly above densest sphere packing. Cell density in a tumor may be not exactly at volume fraction one. Nevertheless, it is very close to volume fraction one, and the available extracellular space is less than in a developing embryo. This result does not mean that density effects can be ignored. Since a growing tumor does not provide the variability to significantly lower cell density, unjamming cannot be controlled by density alone. Nevertheless, the complex interaction between nucleus and cytoplasm may compensate for the lack of extracellular space. The by far softer cytoplasm surrounding a rigid nucleus possibly serves as a kind of intracellular reservoir for free space, which may explain why cancer cells can restore cell motility at such high volume fractions.

This interaction brings cell and nucleus shape into play. Whether in motile cells the shape of cells and nuclei is elongated because they squeeze through the dense microenvironment or their elongated shapes facilitates that the cells can squeeze through is a typical chicken or egg problem. The role of the nucleus in cell jamming is a completely new finding, and, thus, we cannot compare this behavior with any theoretical predictions.

A possibility is that the cell cytoskeleton plays the active part that exerts forces and deforms to unjam in a dense environment, while the nucleus as the mechanically passive part merely mirrors the cellular elongations. In this picture, the nuclear shape variations (also known as pleomorphism) are a reflection of elongated cell shapes, indicating fluidization, and this classical cancer grading criterion would get a reinterpretation as an indicator of the active dynamical state of a tumor.

Strong evidence is provided that shape-induced cell unjamming at high volume fractions exists in 2D. Quantitative agreement is achieved between theoretical simulations based on the idea of a shape-induced unjamming transition at volume fraction one and experiments in cell monolayers $[15,19,23]$. Moreover, medical relevance for asthma and cancer is suggested [1,15]. The physics in 2D cannot per se be applied to 3D. Nevertheless, a recent extension of the self-propelled Voronoi (SPV) model makes precise predictions for the 3D case [52].

The predictions do not seem to be a good match for the actual 3D cell shapes in our investigated spheroids. We do not find a sharp unjamming transition at a predicted critical value for the shape index, since the tissue already solidifies when the cells drastically slow down. An implication of the SPV model $[23,52]$ is that increasing cell-cell adhesion leads to elongation and, thus, to fluidization. However, we have recently found that down-regulation of cadherins fosters unjamming [60], in contrast to the theoretical predictions of the SPV model. The loss of cadherins relates to cancer cell escape and, thus, to a transition where cell density can decrease [60]. The SPV predictions are in 2D monolayers also challenged by the observation that traction, not adhesion, elongates cells [61]. In the system that we investigate here, the fluid MDA-MB-436 cells are also less adhesive than the solidlike MCF-10A cells [40]. This difference indicates that instead of adhesion, e.g., tractions or active contractility could contribute to fluidization in 3D aggregates. Our experimental observations of 3D cell jamming point to more mechanisms - contribution of the nucleus, solidification through a sole slowdown in cell motility, and cell escape regulated by cadherins-which are not included in the current 3D model of shape-induced unjamming. Thus, we also cannot decide if elongated cell as well as nucleus shape are a cause or effect in cancer cell unjamming.

From a biological perspective, shape-induced unjamming makes a lot of sense, since cell elongation, e.g., during polarization, is a basic biological process. Moreover, the recently found differences in cell contractility between epithelial and mesenchymal cells [29,57] favor round 
shapes for healthy cells and elongated shapes for cancer cells. What makes cell aggregates different to conventional particle jamming? Is it simply the rigid cell nucleus surrounded by the soft cytoplasm that brings cells in full contact and still permits that the cells pass by each other? Do cells need to actively move to overcome the yield stress that holds them back? We also cannot ascertain the nature of the transition between the two states, e.g., whether 3D tissues can pass through states with continuously increasing cell motility. Does increased cell-cell adhesion fluidize the system-by increasing traction and elongating cells $[17,52]$ —or solidify the system — by increased intercellular friction [60]? All of the above might play a role. For the development of a tumor, which requires cell motility and proliferation, tissue fluidization through cell unjamming is quintessential. The starting point of cancer requires cell unjamming in a dense microenvironment with less than 5\% free space. This requirement raises open questions about the physics of the process, since it cannot be simply explained by density controlled unjamming.

Our finding that solid tumors have to partially fluidize their tissue to assure proliferation and cell motility is fundamental to tumor biology. In the unjammed tumor areas, where cells behave motile, also cell division is possible since the fluidization rate through cell motility is much faster than through proliferation. It is proposed that elongated cell shape also fosters mitosis [25,35]. For mechanical stability reasons, the fluid tissue fraction cannot exceed the percolation threshold of the jammed areas that form a solid backbone. Nevertheless, since cell motility is a prerequisite for cell escape from the tumor mass that initiates metastasis, it raises the question of how the fluid fraction of cells impacts tumor progression and aggressiveness.

Most cancer markers are prognostic, meaning that they are based on the average of patient outcomes. To avoid over- and undertreatment of patients, oncology needs markers that foresee individual patient outcome. The degree of tumor fluidity in cancer cell clusters as a requirement for tumor growth and metastasis may be such a marker. Tissue fluidity can be measured in vivo by magnetic resonance elastography (MRE) [62]. In averaging over tumor regions, clinical MRE may not detect the fluid tissue areas. For diagnosis, it is irrelevant if cell and nucleus shape are the cause of cancer cell unjamming. Elongated shape and nucleus are a reliable reporter of motile cells and fluid tissues. They can be measured in conventional histological H\&E stained microscopy slides, which are by default taken in standard cancer diagnosis. It has been already empirically shown that grading of the appearance of the nucleus in breast cancer has a certain prognostic power [3]. Our results explain why this grading works. Focused measurements of the cell and nucleus shape index may be an even better individual marker. In synopsis, solid tumors behave like a heterogeneous, active complex fluid, and physical processes and properties are a key determinant of tumor progression. In particular, the mechanics of the cell nucleus plays a critical role not only when cancer cells migrate through the ECM, but also for their motion in cell aggregates.

\section{MATERIALS AND METHODS}

\section{A. Cell and spheroid culture}

MCF-10A cells are cultured in MCF-10A medium: DMEM/Hams F12 medium with L-glutamine (Catalog No. E15-813, PAA Laboratories GmbH, Austria) supplemented with 5\% horse serum (Catalog No. A15-151, PAA), $20 \mathrm{ng} \mathrm{l}^{-1}$ human EGF (Catalog No. E9644, SigmaAldrich), $10 \mu \mathrm{gl}^{-1}$ insulin (Catalog No. I9278, SigmaAldrich), $100 \mathrm{ng} \mathrm{l}^{-1}$ cholera toxin (Catalog No. C8052, Sigma-Aldrich), $500 \mathrm{ng} \mathrm{l}^{-1}$ hydrocortisone (Catalog No. H0888, Sigma-Aldrich), and $100 \mathrm{U} / \mathrm{ml}$ penicillin/streptomycin (Catalog No. P11-010, PAA). MDA-MB-436 cells are cultured in DMEM containing $4.5 \mathrm{gl}^{-1}$ glucose, Lglutamine, but without sodium pyruvate (Catalog No. E15810, PAA) supplemented with $10 \%$ fetal bovine serum (Catalog No. A15-151, PAA) and $100 \mathrm{U} / \mathrm{ml}$ penicillin/ streptomycin. All cell lines are incubated at $37^{\circ} \mathrm{C}$ in a $5 \%$ $\mathrm{CO}_{2}$ atmosphere. The culture medium is changed every 2-3 days, and cells are passaged every 4-5 days. To detach the cells, a phosphate-buffered saline (PBS) solution containing $0.025 \%(\mathrm{wt} / \mathrm{vol})$ trypsin and $0.011 \%(\mathrm{wt} / \mathrm{vol}$ ) ethylenediamine tetra-acetic acid (Catalog No. L11-004, PAA) is applied for several minutes. For spheroid culture, the desired number of cells is placed in nonadherent 96well plates (Catalog No. 781900, BRAND). Spheroids of both cell types are cultivated in 50/50 medium, a mixture of $50 \%$ of the respective media, to ensure that the observed features are cell-type dependent (and, thus, not a mere consequence of medium formulation) and to achieve consistency with our previous publication on spheroid segregation [40]. Both cell types also grow well in $50 / 50$ medium in flasks, and we repeat key experiments of this work in the corresponding "pure" media. MCF-10A spheroids still show jammed characteristics in MCF-10A medium, while MDA-MB-436 spheroids also show the fluid characteristics in MDA medium (data not shown).

\section{B. Fusion assays}

Nonadherent 96-well plates are filled with $150 \mu \mathrm{l}$ of fresh medium, and a pair of spheroids is placed in each well. Spheroids are moved between wells using a $1 \mathrm{ml}$ pipette tip, whose opening is much larger than the spheroids. Spheroids move to the bottom of the wells, where they meet within a few minutes to one hour. Bright-field images of the fusion process are recorded using a Leica $\mathrm{N}$ PLAN $5 X / 0.12$ objective every $5 \mathrm{~min}$ using a CCD camera at cell incubation conditions $\left(37^{\circ} \mathrm{C}, 5 \% \mathrm{CO}_{2}\right)$. 


\section{Live observation of cell motion in spheroids}

Spheroids of 150-200 cells are grown for 1 day in 384well plates with flat microscopy bottom (ibidi), coated with $17 \mu \mathrm{l}$ of $1 \%$ agarose.

Tumors are cut into pieces using scalpels. Tumour pieces are transferred into the same wells and same medium as spheroids.

Images are acquired on a ZEISS Axio Observer spinning disk microscope. We record nuclear vital stain $(0.2 \mu \mathrm{M}$ SiR-DNA, Spirochrome) fluorescence signals every 5 or $7.5 \mathrm{~min}$, for $6 \mathrm{~h}$, sometimes up to $24 \mathrm{~h}$. The deep-red $\left(\Lambda_{e x}=638 \mathrm{~nm}\right)$ illumination minimizes toxicity and is superior in penetration depth, as compared to smaller wavelengths. We use the high-NA water immersion objective ZEISS C-Apochromat 40X/ $1.2 \mathrm{~W}$ to achieve a good signal-to-noise ratio and to match sample and immersion refractive indices. To ensure that the signal does not deteriorate during the observation time, we observe the spheroids in medium supplied with $0.2 \mu \mathrm{M}$ SiR-DNA, which is below the maximum concentration recommended by the vendor of $1 \mu \mathrm{mol}$.

We check that spheroids, even after $24 \mathrm{~h}$ of observation, still fuse, adhere to surfaces, and show cell divisions and cell motility, to ensure that the cells spheroids' biomechanical behavior is not altered by the observation. After observation, the data are registered using a selfwritten MATLAB code (details in the Appendix).

\section{Optical clearing and fluorescence imaging of fixed spheroids}

Spheroids are fixed using 4\% [wt/vol] paraformaldehyde in PBS for $30 \mathrm{~min}$, then consecutively washed using PBS, transferred to a solution consisting of $1 \%$ Triton-X and $1 \% \mathrm{BSA}$ for at least $2 \mathrm{~h}$, then washed using PBS, and then stained using $0.1 \mu \mathrm{M}$ SiR-DNA (Spirochrome) and Alexa-Fluor 488 Phalloidin (Thermo-Fisher, Waltham) for about $24 \mathrm{~h}$.

For optical clearing, spheroids are placed in ibidi IMM mounting medium (ibidi, Munich, Germany) whose refractive index (RI) is measured using a refractometer: $n \approx 1.445$, or in glycerol-water mixtures of corresponding RI. Fluorescence images are acquired on Leica TCS SP2 and TCS SP5 confocal microscopes, using different objectives and immersion media. We investigate how objective resolution, index mismatch between clearing and immersion medium, noise, and other factors affect our results and devote a section of the Appendix to that information. Results shown in Fig. 6 are imaged using Leica HC PL APO 20X/0.7 CS Corr IMM objective with a glycerol-water mixture $(n=1.45)$ as immersion, avoiding any RI mismatch between sample and immersion medium. Confocal sections shown in Fig. 2(a) are recorded using a Leica HC PL APO 63x/1.30 GLYC CORR CS2 objective.

\section{ACKNOWLEDGMENTS}

This project has received funding from the European Research Council (ERC-741350/HoldCancerBack), the Deutsche Forschungsgemeinschaft (DFG KA 1116/17-1 and INST 268/296-1 FUGG), the National Science Foundation (NSF-PHY-1607416), and the Simons Foundation (Grants No. 446222 and No. 454947). We thank Corey O'Hern for helpful discussions. We thank Bernd Kohlstrunk and Barbara Goettgens for technical support.

The authors declare no competing interests.

S. G. contributed fusion analysis, live cell observation and analysis, fixed spheroid image stacks, and analysis of segmentation data. J. L. contributed the 3D-segmentation algorithm. L. O. contributed fusion experiments and analysis and fixed spheroids image stacks. B. A. and S. B. performed surgery. B.W. provided clinical data. E. W. M. and F. R. treated and imaged primary tissue. P. G. and J. L. developed nuclei analysis. T. F. and X. X. measured cell spheroids in the AFM. A. W.F. and S.P. contributed fusion experiments. M. M., D. M. S., J. L., and S. G. developed the shape analysis. J. A. K., M. L. M., B. A., and L.-C.H. designed the research. S. G., J.L., L.O., M.M., D.M.S., M.L.M., and J.A.K. wrote the manuscript. J. A. K. initiated and oversaw the entire project.

\section{APPENDIX: ANALYSIS OF SPHEROID FUSION EXPERIMENTS}

Extraction of the fusion coordinate $\Theta$ is done using a self-written MATLAB fitting routine: We binarize the images using the MATLAB function graythresh. The typical dumbbell shape of fusing spheroids can be approximated by two spherical caps with radius $R$ [42]. We fit, for each frame, the whole $\Theta$-dependent dumbbell shape to the binarized images, finding the pair $(R, \Theta)$ with maximum overlap to the experimental shapes.

This way, we take the whole fusing spheroid shape into account instead of just the growing neck between spheroids. From our experience, the latter measure is disadvantageous, as it is prone to artifacts, and, for later stages of fusion, the neck disappears completely.

Representative results of our fitting procedure are shown in Fig. 2(c).

\section{4D image registration}

We want to observe the spheroids without contact to the substrate or ECM. On nonadherent substrates, they float in medium and are, thus, subject to Brownian motion. With observation times of several hours, it is impossible to assess cell motion without stabilizing (registering) the videos.

We do this image registration using MATLAB function imregister. This function offers two different optimization algorithms, RegularStepGradientDescent (RSGD) and OnePlusOneEvolutionary (OPOE), and two different 
(a) 2018-10-30 436 h24 7.5min-24h - 2: algorithm comparison (downscaled stack)

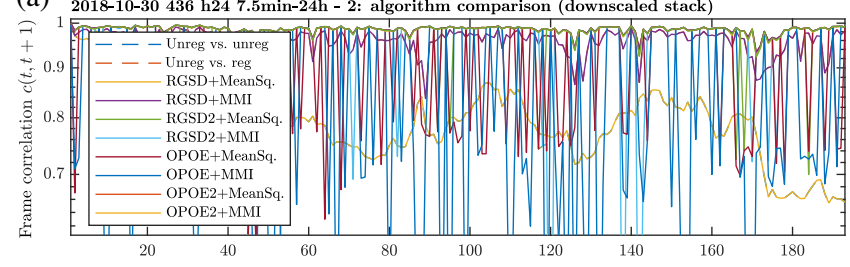

(b) Apply best algorithm to full-size stack

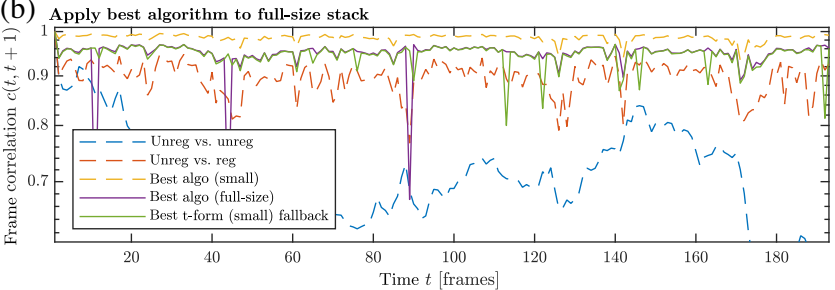

(c) Temporal autocorrelation

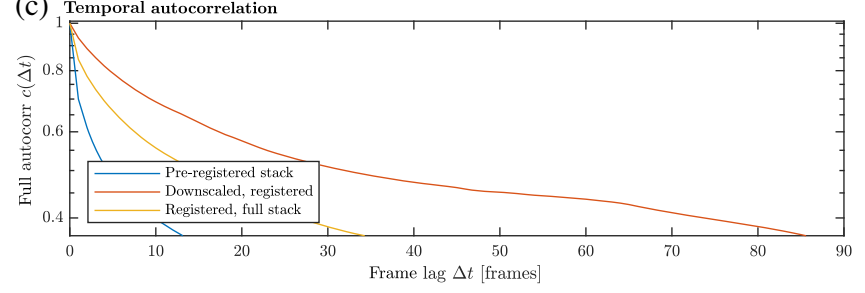

FIG. 8. Multiscale, multialgorithm 3D-registration process. (a) Different optimizers and measures run framewise compared against each other. (b) For each time step individually, the bestperforming algorithm is applied to the full-size 4D image. (c) Registered stacks have much longer signal autocorrelation times than unregistered stacks.

measures of stack overlap, MeanSquares (MeanSq) and MattesMutualInformation (MMI). To measure how successful a registration step is, we calculate the frame-toframe signal correlation between consecutive time steps $c(t, t+1)$ :

$$
c(t, t+1)=\int_{V} s(t) s(t+1) d V .
$$

Here, $s(t)$ is the fluorescence signal. As the cell movements happen on a much slower timescale (hours) than our imaging rate (minutes), the frame-to-frame correlation for successfully registered stacks is well above $90 \%$ (Fig. 8, top).

The algorithm that we apply to each time series of $3 \mathrm{D}$ stacks consists of several steps.

(i) In a first step, the center-of-mass translation of the spheroid is removed. After that, we automatically crop the stacks by thresholding and removing outer (empty) regions.

(ii) We then calculate a down-scaled version of the image stacks with a voxel size of $4 \times 4 \times 4 \mu \mathrm{m}$. We apply all combinations of optimizers (RSGD/OPOE) and measures (MeanSq/MMI) and different sets of parameter settings to each of these down-scaled stacks. This step is done to find out for every time step which algorithm yields the best stabilizing result as measured by maximizing the frame-toframe signal correlation $c(t, t+1)$. Trying it out on a down-scaled version allows us to save time. The frame-to-frame correlations $c(t, t+1)$ for the different sets of algorithms are shown in Fig. 8 (top).

(iii) We apply the best optimizer-measure combination to the full-size stack. It sometimes happens that this combination successfully registers the downscaled stack but fails on the full-size stacks (Fig. 8, middle). In this case, the transformation (T-Form) that successfully worked on the down-scaled stacks is simply used for the full-size stack as well ["best T-Form (small) fallback" in Fig. 8, middle].

As a result, we obtain for each cell type more than five stabilized stacks. Example videos of stabilized stacks are given as Supplemental Material [38].

\section{3D-segmentation procedure}

The prerequisite for this segmentation process are 3D confocal image stacks of cells with stained nuclei and information about the border of the cells. We use an actin stain to obtain the actin cortex as border information and a nuclei stain for center information of the cells. We watershed the actin signal around wells obtained by the nuclei signal. A decent image quality and a low distance between the $z$ stacks are required for good results of the segmentation. The image analysis is done using a selfwritten MATLAB algorithm (Fig. 9).

As a first step, the impact of a lower fluorescence level at the upper parts of the spheroids is reduced by subtracting a morphologically opened image and adjusting the intensity afterward. An adaptive median filter is used to reduce salt and pepper noise. The intensity of dark $z$ slices is increased to match the mean intensity of brighter $z$ slices.

The cell-free background needs to be detected, in order to effectively analyze the border cells of the spheroid. The background detection is done using an entropy filter with the actin signal and smoothing it with a Gaussian filter using a wide kernel. This signal is thresholded, and possible holes are filled.

A good nuclei detection is the most important step in this cell shape segmentation. If multiple nuclei are registered as a single one, their cell volume will be combined, and vice versa, if one nucleus is detected as multiple nuclei, the cell volume will be split. Adaptive thresholding is used on the nuclei signal. In order to minimize false positives, the detected foreground has to be above the threshold of multiple window sizes. The foreground is smoothed by morphological dilation and erosion, holes are filled, and connected regions that are too small to be cell nuclei are deleted.

In this process, nuclei close together are often not segmented but detected as one. Typically, one has ellipsoids which are connected only at a small neck. In order to segment them, the distance of the detected foreground pixels to the background is calculated and smoothed, and its maxima are found [63]. 


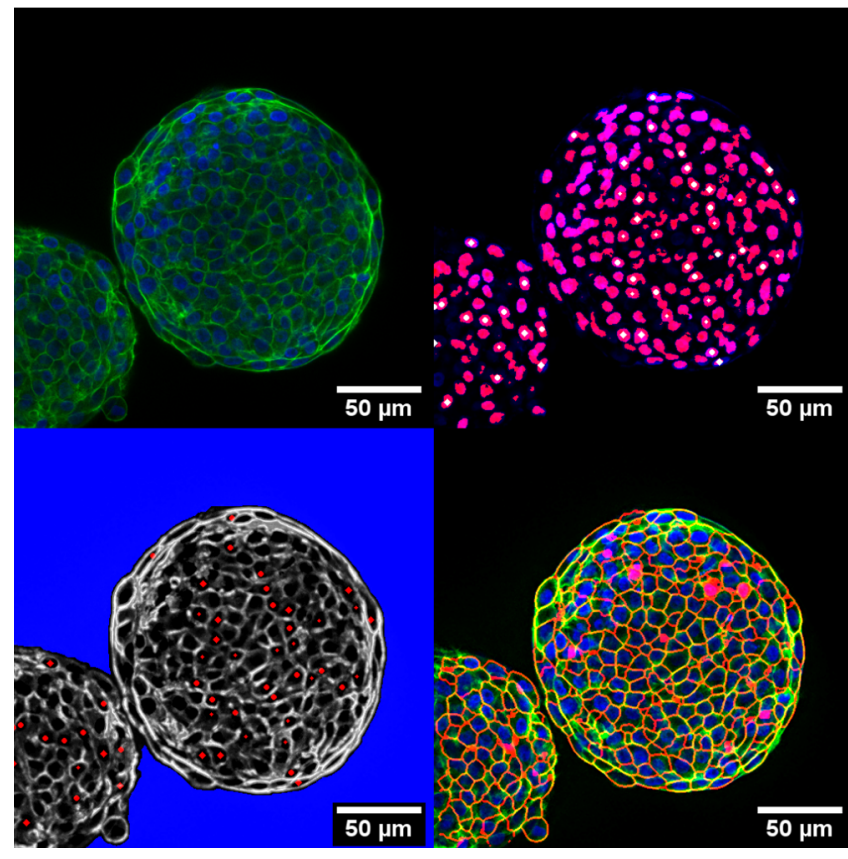

FIG. 9. Illustration of the segmentation process on a sample $z$ slice. Top left: Original data with the actin channel in green and the nuclei channel in blue. Top right: Segmentation of the nuclei, where the nuclei signal is blue and the detected nuclei foreground is red. Cleaned seeds for cell shape segmentation are white. As the seeds are small, most of the seeds of the visible cells are located in nearby planes. Bottom left: Map used for watershedding, based on actin and nucleus signal and the gradient map of the actin signal. The spheroid-free background and the nuclei seeds are false colored blue and red, respectively. Bottom right: Postprocessed actin and nuclei signal in green and blue, respectively, and the outlines of the resulting cell segmentation in red.

These maxima are the future seeds of the cell segmentation. They are dilated with spheres smaller than the typical nuclei radius in order to fuse seeds; if mistakenly, multiple seeds are detected in one nuclei. The dilating sphere is small enough that seeds of different nuclei almost never fuse.

The segmentation is done on a gradient map of the actin signal. This gradient map is smoothed with a small Gaussian kernel to avoid two different maxima lines on the border of actin strands. Afterward, the gradient map is superimposed with zeros on the location of the nuclei seeds and the previously detected cell-free background. This image is used as the basis for watershedding.

The detected watershed lines form a connected network of cell borders. Usually, some misdetections are present, which are identifiable by their small size, usually much smaller than a typical size, and by not containing any nuclear seeds. Those misdetections are deleted and their volumes added to the neighboring outlines. Afterward, the outlines are shrunk again. An additional step to increase segmentation quality is to check which seeds lead to misdirected shapes, sort those out, and rerun the segmentation.

\section{a. Influence of image quality and noise on segmentation}

To test the influence of the image quality on the segmentation results, we artificially degrade the image quality of a control spheroid stack by a combination of two methods. The first is smoothing the data with a 3D Gaussian kernel, with a standard deviation of $\sigma$. We vary $\sigma$ up to $\sigma=1.5 \mathrm{px}(1 \mathrm{px} \approx 600 \mathrm{~nm})$, which means this maximum smoothing width is larger than the lateral optical resolution $\left(d_{x} \approx 0.6 \lambda / \mathrm{NA} \approx 500 \mathrm{~nm}\right)$, causing visible smoothing of data. Second, we also add artificial noise by adding a zero-mean, Gaussian white noise with strength noise. We vary noise up to a relative intensity of 0.1 , introducing noise that is much larger than the original noise in the data. We run the segmentation on a total of 42 of these artificially degraded image stacks (seven different sigma values and six different noise values).

We observe that, when we combine both effects at their maximum strength, the segmentation visibly degrades (Fig. 10), although large parts still stay intact. However, under all conditions, the core-shell structure of MCF-10A spheroids, meaning lower cell shapes in the center and higher, elongated cells at the rim, is stable under these modifications (Fig. 11). Additionally, there is no clear trend that drives up (or down) cell shapes, in neither the spheroid core nor the shell.

We conclude that the cell shape indices are not an artifact of the image acquisition process.
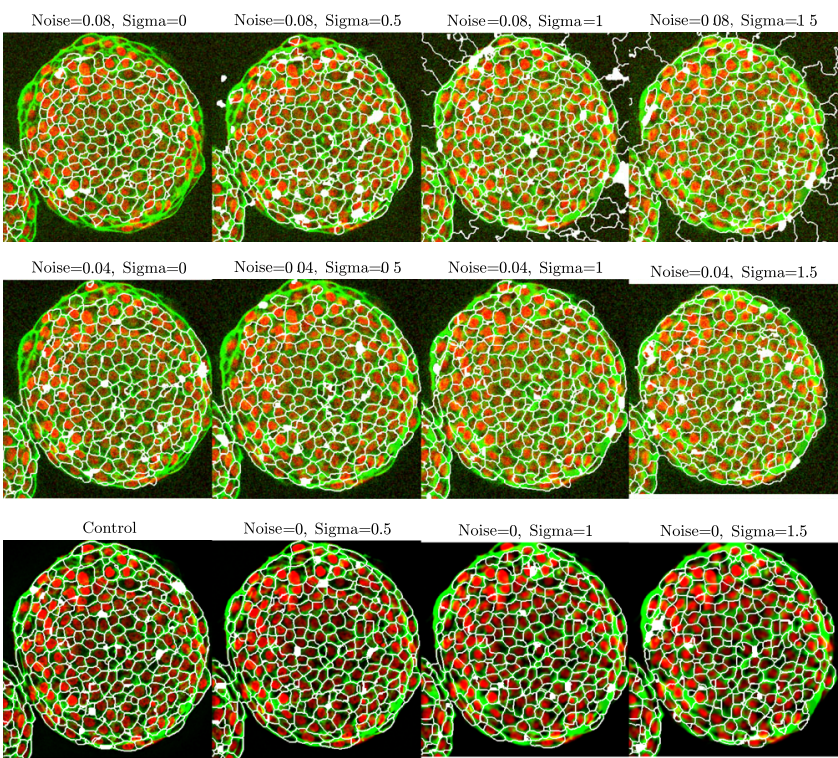

FIG. 10. 3D segmentation with artificial noise and smoothing. We add Gaussian white noise to a fluorescent image stack and smooth it such that the image quality is severely deteriorated. We compare the results of the 3D-segmentation algorithm in Fig. 11. 

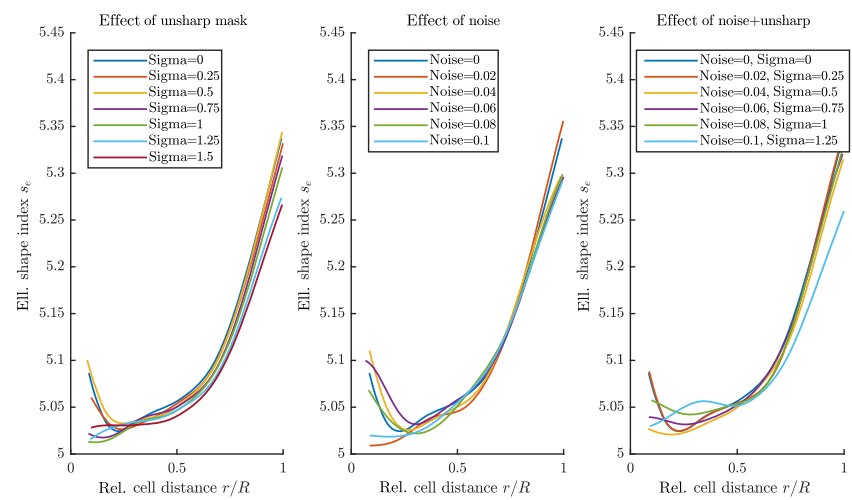

FIG. 11. The cell shape distribution within the spheroids is stable under severe artificial degradation of image quality. Shown is the ellipsoid shape index $s_{E}=S_{V} V_{V}^{-2 / 3}$ [which is the same as in Eq. (3) but without the normalization by the constant factor $\left.(36 \pi)^{-1 / 3}\right]$.

\section{b. Influence of refractive index mismatch between mounting and immersion medium}

Any mismatch between the objective immersion refractive index (RI) $n_{1}$ and the RI of the sample mounting medium $n_{2}$ introduces optical aberrations that distort the confocal image stacks [53] in the $z$ direction. These have to be taken into account in order to reliably quantify $3 \mathrm{D}$ cell shapes.

Theoretical and experimental results lead to a $z$-scaling factor of [53]

$$
z^{*}=\frac{\mathrm{AFP}}{\mathrm{NFP}}=n_{1} / n_{2}
$$

Here, AFP and NFP refer to the actual vs nominal focus points, respectively, as defined in Ref. [53].

We embed spheroids in $72 \pm 15 \%$ wt. glycerol, yielding a refractive index of the mounting medium $n_{2}=1.431 \pm$ 0.024 . The uncertainty stems from the fact that one could not always remove remaining medium around spheroids. As a second uncertainty source, glycerol-water mixtures change their composition and, thus, their RI over time, depending on the humidity. As immersion, we use preferably glycerol with $n_{1}=1.47$, but sometimes also water with $n_{1}=1.33$, for example, when the objective specifications require it. This difference leaves us with an uncertainty in the $z$-scaling factor.

To investigate how the $z$ distortion affects the cell shape index, we numerically vary the scaling factor $z^{*}$ for each spheroid (Fig. 12) over a certain range. We find that, in the proximity of the theoretical scaling factor $z^{*}$, cell shape indices typically acquire a minimum value (Fig. 12). Most importantly, the uncertainty in the scaling factor $z^{*}$ is by far not large enough to influence the measured cell shape results.

In a second set of measurements, we mount and clear spheroids in ibidi IMM mounting solution, which is

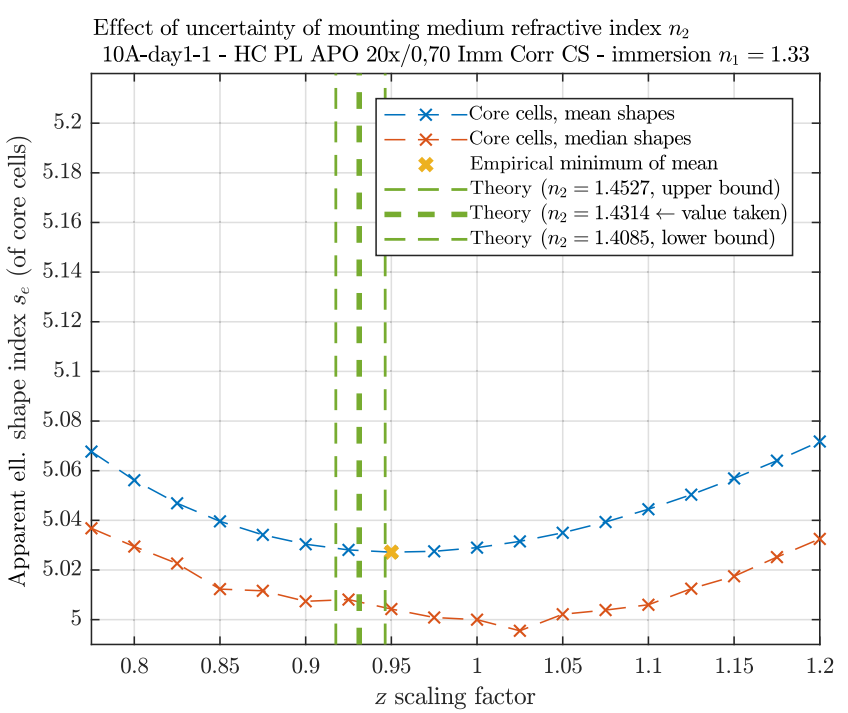

FIG. 12. Variation of the scaling factor $z$. The theoretical prediction for $z$-scaling factor from Ref. [53] minimizes average cell shape indices in the core of the spheroid. Shown are data for an MCF-10A spheroid.

glycerol-based and supplied with antifade agents. As glycerol-water mixtures change their RI depending on the relative humidity of the environment, we measure the RI of the mounting solution using a refractometer. We then use a glycerol-water mixture with an RI adjusted to that of the mounting medium as objective immersion, thus matching the two RIs as good as possible. The cell shape indices measured by this method yield results which are not discernable from the first method, for both MCF-10A and MDA-Mb-436 spheroids.

\section{Segmentation of nuclei in primary human tumor}

The data for the method described hereafter are static images from a representative $z$ plane and time $t$ from videos of live observations of manually dissected pieces of tumors stained with a vital DNA stain. The image is analyzed with the FIJ [39] plugin StarDist [64], which is a nuclei detection algorithm based on star-convex polygons. Nuclei which are falsely segmented are manually corrected. Moving and nonmoving areas are visually assessed by evaluating the movies prior to the segmentation.

\section{Cell shape quantification}

To calculate the ellipsoid cell shape factor $s_{E}$ [Eq. (3)], we compute the second volume moment $J_{i j}$ of the cell volume (with $i$ and $j$ being dimension indices). This moment $J_{i j}$ is closely related to the cell moment of inertia and is defined as

$$
J_{i j}=\int_{V}\left(r_{i}-r_{i}^{\mathrm{cm}}\right)\left(r_{j}-c_{j}^{\mathrm{cm}}\right) d^{3} r .
$$


Here, the integral is over the volume $V$ of the cell, $\vec{r}$ is the position within the cell, and $\vec{r}^{\mathrm{cm}}$ is the cell center of mass.

For an ellipsoid with half axes $a>b>c$, oriented along the $x, y, z$ axes, respectively, one obtains the following second moment $J_{i j}^{E}$ :

$J_{x x}^{E}=\frac{1}{5} V_{E} a^{2}, \quad J_{y y}^{E}=\frac{1}{5} V_{E} b^{2}, \quad J_{z z}^{E}=\frac{1}{5} V_{E} c^{2}$,

and the nondiagonal elements zero, where $V_{E}=$ $(4 \pi / 3) a b c$ is the volume of the ellipsoid.

Thus, to find the corresponding ellipsoid for a given cell, we compute the cell segment's second moment tensor $J_{i j}$ and look for the ellipsoid with the same second moment tensor. To this end, we diagonalize $J_{i j}$ and compare the resulting eigenvalues $J_{1}, J_{2}$, and $J_{3}$ to Eq. (A4) to extract the volume $V_{E}$ and the half axes $a, b, c$ of the fitted ellipsoid:

$V_{E}=\left(\frac{2000 \pi^{2} J_{1} J_{2} J_{3}}{9}\right)^{1 / 5}, \quad a=\left(5 J_{1} / V_{E}\right)^{1 / 2}$, etc.

To compute the ellipsoid surface, we use the approximation formula:

$$
S_{E}=4 \pi / 3\left[(a b)^{8 / 5}+(a c)^{8 / 5}+(c b)^{8 / 5}\right]^{5 / 8} .
$$

$S_{E}$ and $V_{E}$ yield the dimensionless ellipsoid shape index $s_{E}$ [Eq. (3)].

To compare these values to the jamming threshold proposed in Ref. [52], we apply the same rationale to the Voronoi cells in the simulation. The jamming threshold value is less sharp in this measure and lies near $S_{E}^{*} V_{E}^{*-2 / 3} \approx 4.85 \ldots 4.90$. Normalizing by the sphere shape factor $\sqrt{36 \pi}$ as in the main manuscript, this value yields a threshold $s_{E}^{*}=1.003-1.013$.

\section{Comparison and discussion of $3 \mathrm{D}$ cell shape measures}

In the main text, we use the ellipsoid shape index $s_{E}$ as a measure to characterize cell elongation. Other measures are also suitable, each having their own advantages and disadvantages. Among them are the Voronoi cell shape index $s_{V}$, the cell aspect ratio $\mathrm{AR}$, and the raw surface-tovolume ratio $s$.

(i) The ellipsoid shape index $s_{E}$ is used in the main manuscript. It relies on the second moment tensor of a volume segment, is, thus, independent of the neighbor cells (unlike $s_{V}$ ), and can be defined for all cells in a spheroid individually, even for boundary cells. The second moment tensor representation, similar to inertia tensors, is an integral over the complete volume and is, thus, relatively insensitive to the exact shape of the surface of a segment (as the actual surface-to-volume ratio $s$ is).
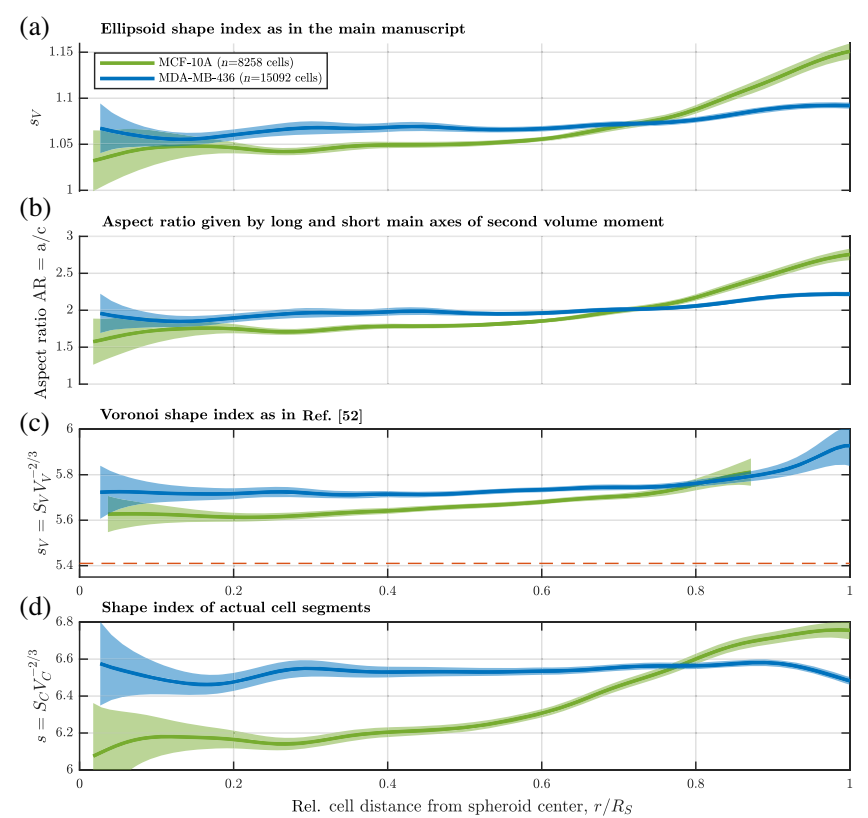

FIG. 13. Comparison of different shape measures. (a) Ellipsoid shape index $s_{E}$, as in the main manuscript. Shaded areas are confidence intervals of moving bins of FWHM $=0.1 R_{S}$. (b) Cell aspect ratio $\mathrm{AR}=a / c$, where $a$ is the longest and $c$ the shortest axis of the ellipsoid of corresponding second volume moment of the cell. (c) Shape index of Voronoi cells constructed from the barycenters of the cell segments found by the segmentation, analog to the simulation in Ref. [52]. (d) Shape index $s$ of the actual, raw segments.

It is defined in the main manuscript. Note that we use a normalized version which, for spherical cells, yields $s_{E}=1$. The normalization factor $(36 \pi)^{1 / 3} \approx$ 4.76, which would otherwise appear as base index for spherical cells, can confuse any reader who is not familiar with SPV models, and we would like to address a broader physics and biology audience.

The values for both spheroid types are shown in Fig. 13.

(ii) The Voronoi shape index $s_{V}$ is used in a simulation of the 3D SPV model [52]. In this simulation, there are no boundary cells (periodic boundary conditions). A disadvantage of this measure is that Voronoi cells are defined by their neighbors, so that, in our experimental study, cells near the rim of the spheroid are undefined. This shape measure thus cannot fully capture the increased cell elongation of the outer cell layers.

We use the barycenters $\vec{r}^{\mathrm{cm}}$ of the cell segments as input to a 3D-Voronoi tessellation and compute the Voronoi shape index $s_{V}$ :

$$
s_{V}=S_{V} V_{V}^{-2 / 3} .
$$

Nonetheless, cell shapes in this measure can be compared to theory predictions. Cell shapes that we 
find within the solidlike MCF-10A spheroids lie above the jamming threshold value of $s_{V}^{*} \approx 5.4$ reported in Ref. [52], just as for the $s_{E}$ measure.

The values for both spheroid types are shown in Fig. 13.

(iii) The aspect ratio AR is used in, e.g., Ref. [19]. It also relies on the ellipsoids of same second volume moment, just as $s_{E}$, is, thus, also relatively insensitive to the exact shape of the surface, and can be defined for all cells, including boundary cells.

In 2D studies, such as Ref. [19], AR is simply given by the relation of long and short axes of the ellipsoid: $\mathrm{AR}=a / b$ (for $a>b$ ). In a 3D system however, cells have three main axes. We define the aspect ratio by the ratio of the longest to the shortest of the three axes $a, b, c$ (where $a>b>c$ ), ignoring $b$ :

$$
\mathrm{AR}=\frac{a}{c} .
$$

An immediate advantage is that aspect ratio values are intuitive; e.g., one can imagine a 3D volume with $\mathrm{AR} \approx 2$. However, by ignoring the main axis $b$, some information is lost. The aspect ratio nonetheless leads to the same conclusions, e.g., MDA-MB-436 cells being significantly more elongated, except for MCF-10A cells near the boundaries.

The values for both spheroid types are shown in Fig. 13.

(iv) The raw, actual surface-to-volume ratio $s$ is defined in analogy to $s_{E}$ and $s_{V}$ but relies on neither Voronoi tessellation nor second moment representation of the segments. Instead, the raw segment surface $S$ and volume $V$ are used:

$$
s=S V^{-2 / 3} .
$$

This definition makes this measure relatively sensitive to surface undulations, or wrinkled cell boundaries, as wrinkles immediately drive up the surface $S$ but not the volume $V$. A wrinkled surface increases $s$ and, thus, has the same consequences as overall cell elongation. This effect is the reason why the $s$ values are generally higher than, e.g., $s_{V}$ values (Fig. 13).

Nonetheless, in this measure as in any other measure, MDA-MB-436 cells have a significantly higher shape index than MCF-10A (except for boundary cells).

The values for both spheroid types are shown in Fig. 13.

\section{Volume fraction in spheroids}

In recorded high-resolution 3D stacks of the actin and nuclei of the cell spheroids, we do not see spaces between

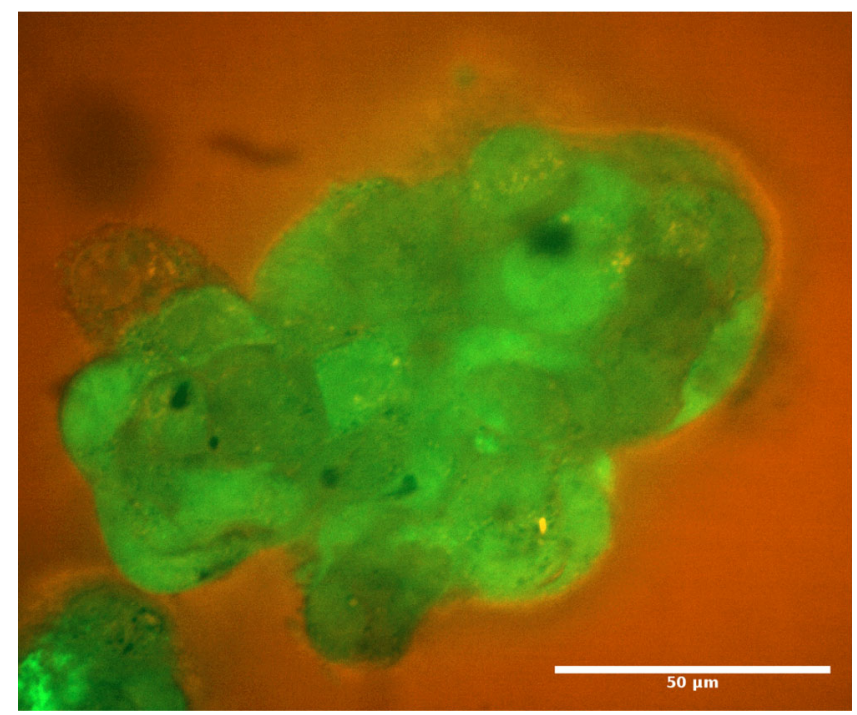

FIG. 14. Example for dense cell clusters with a high volume fraction. MDA-MB-436 spheroid with cytosol stained in green and medium stained in red. The stains show dense aggregates with no apparent spaces between the cells inside the spheroid.

cells in the optical resolution limit. To further evaluate this assumption, interstitial fluid is stained in MDA-MB-436 spheroids (Fig. 14). This technique is already described in Refs. [65,66]. Cells are labeled with $2 \mu \mathrm{M}$ CellTracker Green CMFDA (Invitrogen/Molecular Probes) and $0.1 \mathrm{gl}^{-1}$ Molecular Probes ${ }^{\mathrm{TM}}$ Dextran, Alexa Fluor ${ }^{\mathrm{TM}}$ 64710000 MW (ThermoFisher) for $45 \mathrm{~min}$ and subsequently washed with PBS before they are detached, plated into ultralow adhesion plates to form spheroids, and imaged as described above.

The stains show dense aggregates, and there are no visible interstitial spaces inside the spheroid, which is different from the situation in Refs. [65,66], where considerable amounts of interstitial fluid between cells showed up.

\section{Motility state of primary tissue samples}

Table I gives an overview of primary tumor samples which we investigate. Table II summarizes results sorted for grading, staging, and nodal status. There is no significant correlation between stage, grade, or nodal status to the presence of moving areas (Fisher's exact test, $p=0.05$ ).

\section{AFM measurements on cell spheroids}

The typical curves show a near-perfect fit to the Hertz model with a single elastic modulus over the whole indentation range. A softer or stiffer ECM layer on the surface results in a deviation of the indentation from the Hertz model with a single modulus. A curve with distinct layers could be broken down to individual segments with distinct moduli [67]. For example, a softer surface layer 
TABLE I. Motility state of primary tissue samples. Overview of investigated clinical samples. Grading and staging as given by the pathology; "..." means unknown or not transferred. "m1mic" means micrometastasis. Motility status is detected from live observation as described in the manuscript. "motile" means fluid parts are found, while "resting" means that no motile parts are found in the tissue pieces that are observed, which, however, do not comprise the whole tumor.

\begin{tabular}{lcccc}
\hline \hline Internal ID & Type & Grading & Stage & Motility status \\
\hline 84 & Cervix & 3 & $\ldots$ & Motile \\
86 & Cervix & 3 & 4 & Resting \\
87 & Cervix & $\ldots$ & $\ldots$ & Resting \\
88 & Cervix & $\ldots$ & $\ldots$ & Resting \\
90 & Cervix & 3 & $2 \mathrm{~b}$ & Motile \\
93 & Cervix & 3 & $3 \mathrm{~b}$ & Resting \\
95 & Cervix & $\ldots$ & $\ldots$ & Resting \\
96 & Mamma & 2 & 2 & Motile \\
99 & Cervix & 2 & $2 \mathrm{~b}$ & Motile \\
100 & Mamma & 2 & $1 \mathrm{c}$ & Resting \\
101 & Mamma & 1 & $1 \mathrm{c}$ & Resting \\
103 & Cervix & $\ldots$ & $\ldots$ & Motile \\
104 & Cervix & 3 & $2 \mathrm{~b}$ & Resting \\
105 & Cervix & $\ldots$ & $\ldots$ & Motile \\
106 & Mamma & 2 & m1mic & Motile \\
108 & Mamma & 3 & 3 & Motile \\
\hline \hline
\end{tabular}

leads to a curve that starts out flatter while the soft surface layer is compressed and later changes to a steeper curve as the stiffer underlying layer is compressed. A stiffer surface layer leads to a curve that approaches the simple Hertz fit from below. A typical AFM curve is shown in Fig. 15.

TABLE II. Motility state of primary tissue samples sorted by origin and classification.

\begin{tabular}{lc}
\hline \hline & Moving/Total samples \\
\hline Total & $8 / 16$ \\
Biopsies (cervix) & $1 / 7$ \\
Mesometrial resections (cervix) & $5 / 5$ \\
Mastectomies & $2 / 4$ \\
Grading known & $6 / 11$ \\
Grade 1 & $0 / 1$ \\
Grade 2 & $3 / 4$ \\
Grade 3 & $3 / 6$ \\
Nodal status known & $5 / 9$ \\
Nodal 0 & $4 / 6$ \\
Nodal 1 & $1 / 3$ \\
Histopathologic staging known & $4 / 9$ \\
Stage 1c & $0 / 2$ \\
Stage 2, b & $4 / 5$ \\
Stage 3b & $0 / 1$ \\
Stage 4 & $0 / 1$ \\
\hline \hline
\end{tabular}

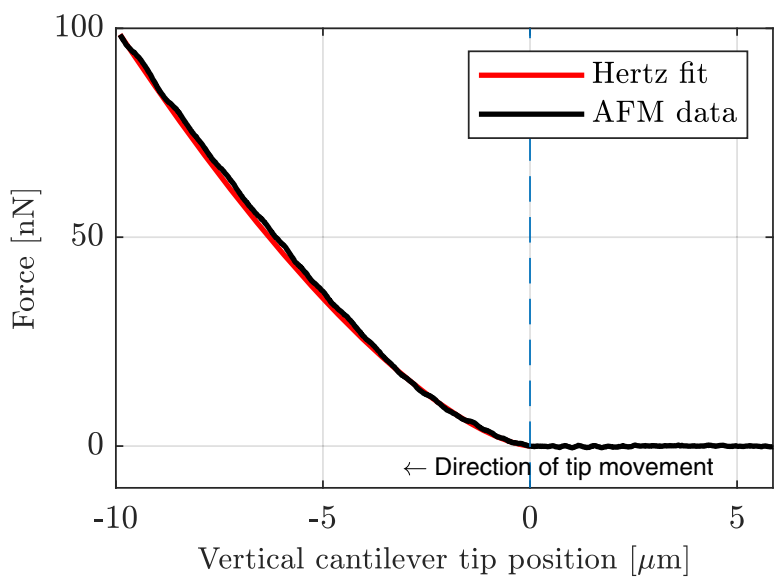

FIG. 15. AFM force vs indentation curves cell aggregates show no signs of ECM. Representative image of a force-indentation curve. Aggregates (this example shows MCF-10A) are probed with a tipless AFM cantilever (TL-Cont-20, Nanosensors, $50 \pm$ $7.5 \mu \mathrm{m}$ cantilever width). Spheroids are indented for several micrometers up to a final indentation force of $100 \mathrm{nN}$. Data (black) are fitted with a Hertz model (red), using the approximation of a small soft sphere indented by a much stiffer, much larger sphere. The vertical position is set to 0 at the contact point (dashed blue line).

\section{Cell-line-specific fusion is independent of spheroid size}

Figure 16 shows that the qualitative fusion behavior (fluidlike or solidlike) is specific for the cell lines but does not depend on spheroid size.
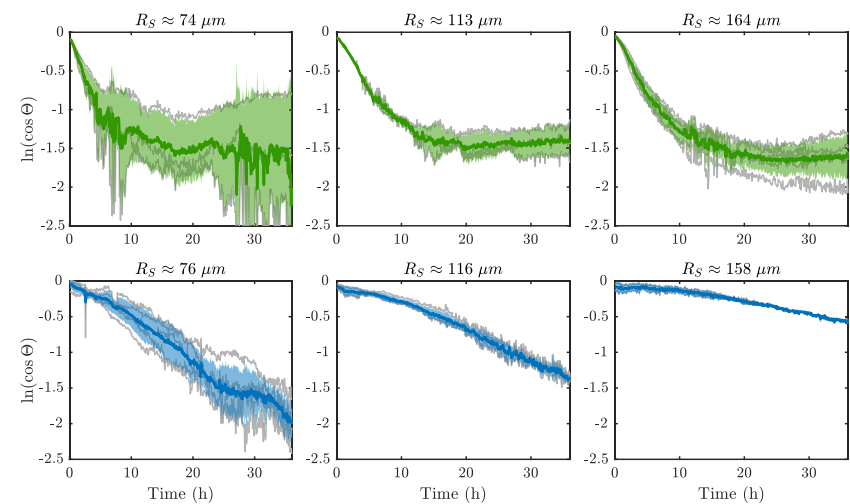

FIG. 16. Cell-line-specific qualitative fusion behavior is independent of spheroid size. Fusion coordinate $\ln \cos \theta$ for an experiment series with spheroids with 500, 2000, and 8000 cells (left to right) and both cell types: MCF-10A (top line) and MDAMB-436 (bottom line). All MCF-10A spheroids display the characteristic arrest, and all MDA-MB-436 fusions show the apparently fluidlike scaling. Every figure comprises at least three experiments (mean + SEM). 


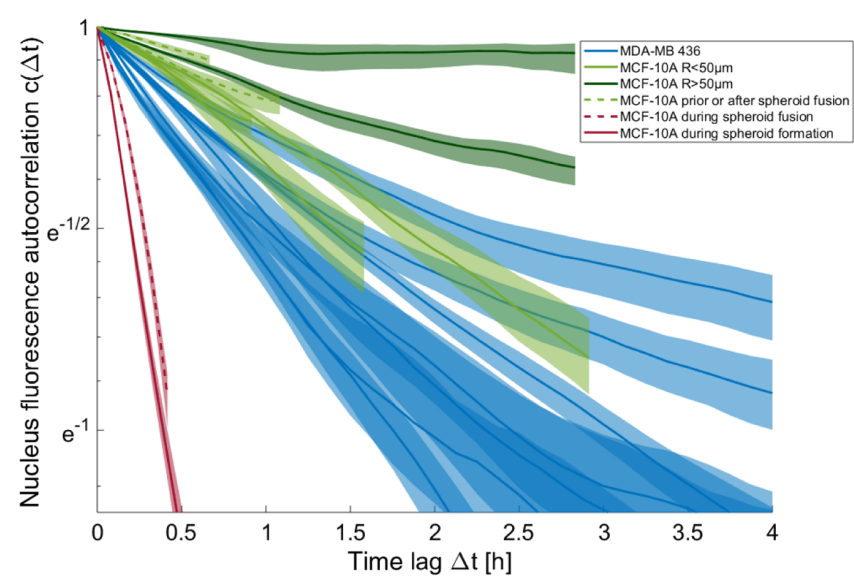

FIG. 17. Temporal autocorrelation of the nucleus signal in registered live tracking experiments of $\mathrm{MCF}-10 \mathrm{~A}$ spheroids in differing conditions and MDA-MB-436 spheroids. The correlation on the $y$ axis is on a logarithmic scale. The bright regions indicate the $90 \%$ confidence interval of the autocorrelation function, estimated by bootstrapping.

\section{3D autocorrelation analysis of live cell motion}

The temporal autocorrelation is calculated from the locations that persist through every time point of the registered stacks using the following equation:

$c(\Delta t)=1 / \sigma(f) *\langle[f(t)-\langle f\rangle] *[f(t+\Delta t)-\langle f\rangle]\rangle$,

where $f(\vec{x}, t)$ is the fluorescence intensity of the pixel of the $3 \mathrm{D}$ registered stack at time $t .\langle f\rangle$ indicates the mean fluorescence intensity of the data, and $\sigma(f)$ is the variance of the intensity signal of the data. The overall mean is taken over all possible locations and time points. For bootstrapping, out of all possible pixels that persist through every time point of the registered stacks, $n$ times a random pixel is drawn (with placing back), where $n$ is the number of possible pixels. This result is used as new input data, and the correlation is calculated as described above. After doing that 100 times, the $90 \%$ confidence interval at each lag time $\Delta t$ is given by the fifth lowest and highest correlation of the bootstrapped data for this lag time. The averaged curve is, correspondingly, the curve of the median values of the bootstrapping process. This process does not change the general behavior, compared to calculating the autocorrelation simply for all pixels. It is chosen because the median curve is more central in the confidence interval and, therefore, gives a better visual impression.

A decay in the autocorrelation of the nucleus signal of registered stacks captures the movement of the nuclei and by proxy the movement of the cells. For MDA-MB-436 spheroids, the decay happens at a very comparable speed and is nearly exponential for most of them. This result is constant with our picture of them, as spheroids fluidized by active motion. Judging from Fig. 17, the single-cell speed in MDA-MB-436 spheroids is relatively independent of the spheroid properties.

The decay in epithelial-like MCF-10A spheroids paints a distinctly different picture. For the large spheroids we observe, the autocorrelation does not decay to less than 0.7 , and 0.9 for one example, within $3 \mathrm{~h}$. This observation demonstrates a distinct lack of motion, consistent with our assessment of them as jammed spheroids. MCF-10A spheroids with a lower radius show a higher amount of internal motion, captured by a faster decay in the temporal nucleus correlation. This trend can be explained by (a) the surface tension having a higher relative effect and ability to fluidize the system overcoming a yield stress and (b) the jammedlike core we observe in the cell segmentation having a lower share of the spheroid volume in smaller spheroids. Interestingly, very fast relatively cell motions can be induced in MCF-10A spheroids by external forces, for example, during the spheroid formation or during spheroid fusion. This result is consistent with the observed nematic orientation of cells MCF-10A during the fusion of their spheroids and with the observed fast starts of the fusion of these spheroids. The autocorrelation shows a typical decay before and after the fusion event, indicating that there are no fundamental changes in the cells during these events that persist.

\section{Examples of full spheroid segmentation analysis}

For a total of more than 20 spheroids of the MDA-MB436 and MCF-10A type of different ages, all comprised of typically between 1000 and 2000 cells, we create overview sheets as shown in Fig. 18. This overview is done in order to ensure the quality of the segmentation of all spheroids included in the analysis.

\section{Testing the segmentation on synthetic 3D data with known shapes}

Two artificial pseudofluorescence stacks made of actin and DNA signals are used to test the segmentation algorithm. One simulated spheroid is made of relatively roundish cells, and one has more elongated cells. In both cases, the test cell shapes are known and can, thus, be compared to the segmentation results. No adjustments are made in the segmentation algorithm, with the sole exception that mat files with artificial, simulated data are loaded rather than microscopy data. The stacks are realistic and challenging with respect to several aspects.

(i) Nuclei have different sizes (varying by a factor of 2 in each dimension) and different brightness.

(ii) One sample has more elongated nuclei than the other one.

(iii) Nuclei are excentric with respect to the segment barycenters and sometimes overlap with the actin boundaries and even with neighboring nuclei. 


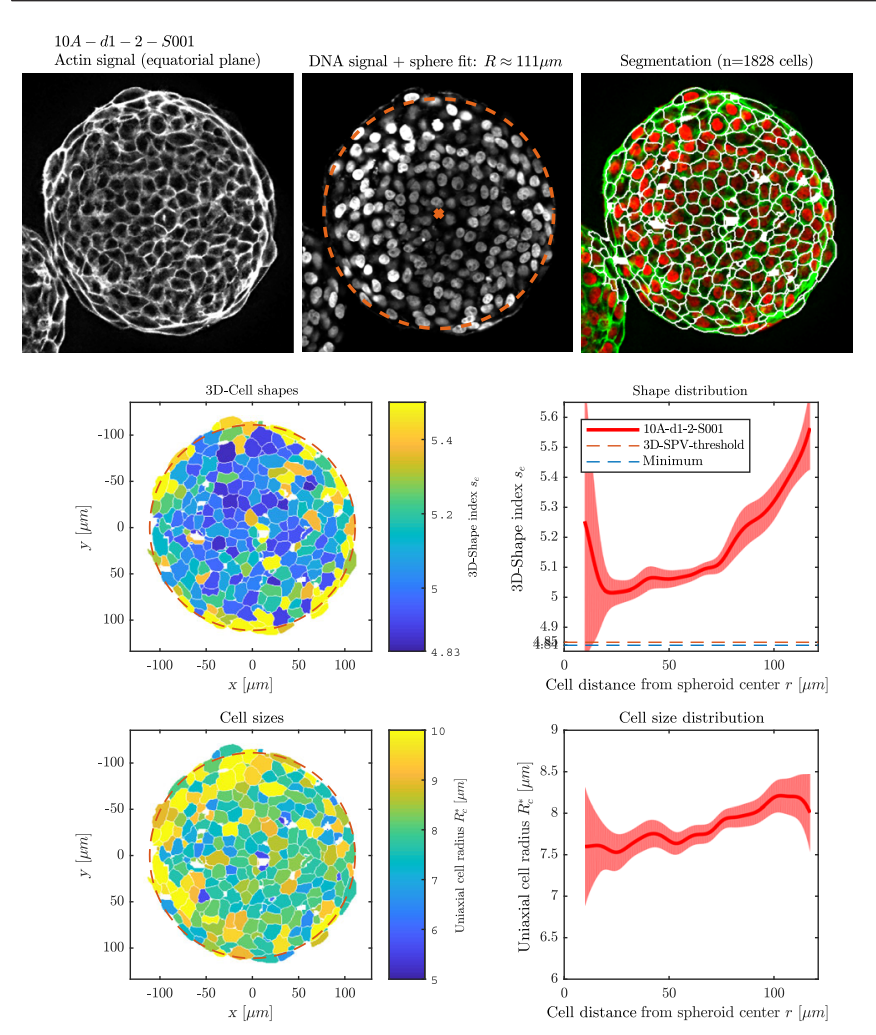

FIG. 18. Example of a MCF-10A spheroid segmentation overview figure. Actin/DNA signals are the confocal signals, with global contrast adjusted using MATLAB's imadjust function to ensure good visibility. Shown is the ellipsoid shape index $s_{E}=S_{V} V_{V}^{-2 / 3}$, which is the same as in the main manuscript, except for the missing normalization by the constant factor $(36 \pi)^{-1 / 3} \approx 4.836$.

This overlap does not happen in nature, but it is present in our confocal microscopy data, as optical resolution is limited.

(iv) There is more noise in the synthetic data than in actual confocal microscopy stacks.

As shown in Fig. 19, the algorithm accurately captures cell boundaries and shapes, and the detected cell shapes are very close to the known input shapes. Furthermore, there is a movie of the spheroid segmentation presented in Supplement Material [38], allowing the reader to assess the segmentation quality by eye.

The shape distributions can clearly discern between the two artificial spheroids. This distinction is shown by a statistical analysis comparing the two artificial spheroids.

One has mean shape $s_{E}^{\text {input }}=1.018$; the segmentation finds mean shape factor of $s_{E}^{\text {detected }}=1.015$. The second, more elongated, has $s_{E}^{\text {input }}=1.037$; here, the segmentation finds $s_{E}^{\text {detected }}=1.035$. For both spheroids, the difference between input shape distribution and the segmented shape distribution is not statistically significant (two-sided Kolmogorov-Smirnov test, $p=0.08$ and $p=0.16$, respectively), as the detected shapes are close to input shapes.
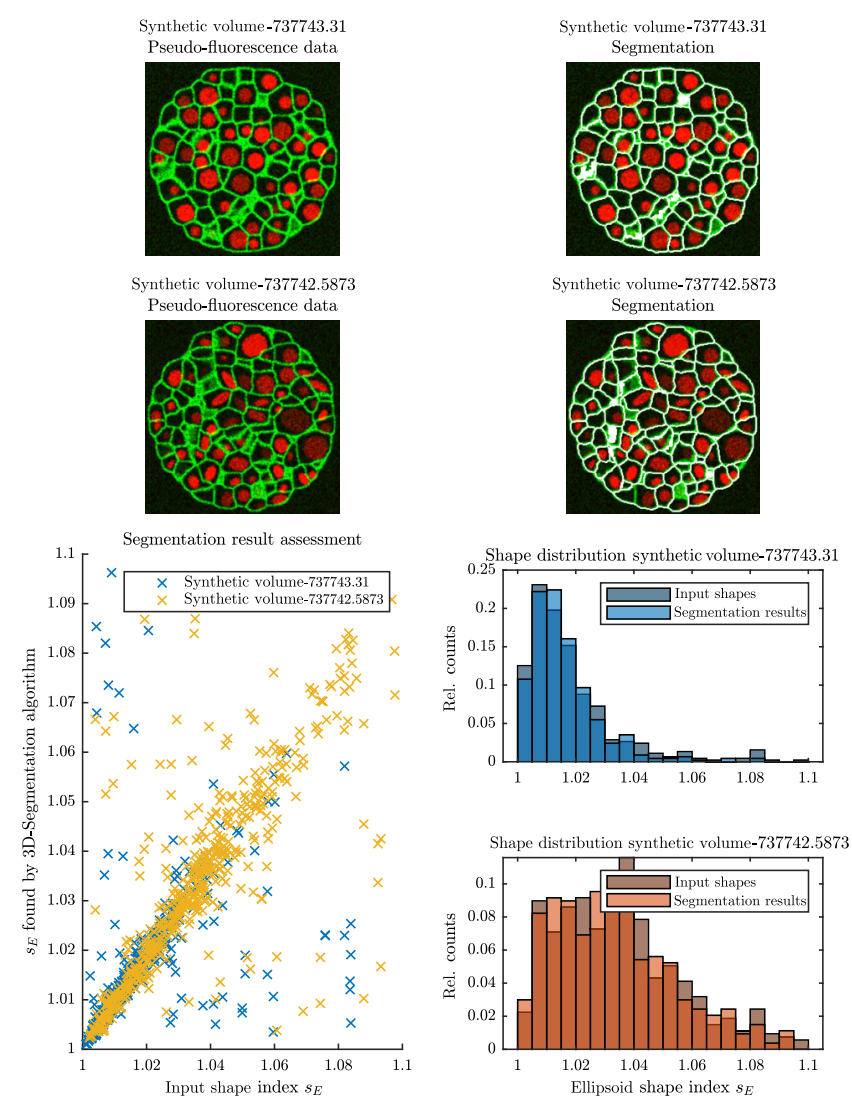

FIG. 19. Two different artificial 3D volumes of pseudofluorescence data are used to test the automatic 3D segmentation, one with more roundish and one with more elongated cell shapes. Cell shapes in these test volumes are known. The figure shows equatorial slices through the volumes and the segmentation results. The shape distribution histograms for both stacks overlap with the actual input shape distributions.

However, the known input distributions are significantly different between the two spheroids $\left(p<10^{-43}\right)$; also, the distributions detected by the segmentation algorithm are significantly different $\left(p<10^{-50}\right)$, meaning that the algorithm can discern between the different spheroids.

The mean shape factor difference between the two spheroids is $\Delta s_{E} \approx 0.02$, which is roughly comparable to the difference measured between MCF-10A and MDAMB-436 spheroid types. In any case, the error in detected mean shape factor $s_{E}^{\text {input }}-s_{E}^{\text {detected }}<0.003$ is considerably smaller than the difference between the actual spheroids as such, $\quad s_{E}^{\text {input }}-s_{E}^{\text {detected }} \approx 0.003<\Delta s_{E} \approx 0.02$. In other words, the error is much smaller than the signal. This result underlines that the algorithm can reliably discern between these two spheroids.

These artificial spheroids consist of about 500 cells each. The real spheroid data in the main manuscript consist many thousands of cells for each type, which makes shape differences statistically easier to detect. 
[1] L. Oswald, S. Grosser, D. M. Smith, and J. A. Käs, Jamming Transitions in Cancer, J. Phys. D 50, 483001 (2017).

[2] H. J. G. Bloom and W. W. Richardson, Histological Grading and Prognosis in Breast Cancer, Br. J. Cancer 11, 359 (1957).

[3] W. H. Wolberg, W. N. Street, and O. L. Mangasarian, Importance of Nuclear Morphology in Breast Cancer Prognosis, Clin. Cancer Res. 5, 3542 (1999), https:// pubmed.ncbi.nlm.nih.gov/10589770/.

[4] J. S. Meyer, C. Alvarez, C. Milikowski, N. Olson, I. Russo, J. Russo, A. Glass, B. A. Zehnbauer, K. Lister, R. Parwaresch, and f.t. C. B. C. T. Resource, Breast Carcinoma Malignancy Grading by Bloom-Richardson System vs Proliferation Index: Reproducibility of Grade and Advantages of Proliferation Index, Modern pathology 18, 1067 (2005).

[5] E. Jonietz, Mechanics: The Forces of Cancer, Nature (London) 491, S56 (2012).

[6] A. Fritsch, M. Höckel, T. Kiessling, K. D. Nnetu, F. Wetzel, M. Zink, and J.A. Käs, Are Biomechanical Changes Necessary for Tumour Progression?, Nat. Phys. 6, 730 (2010).

[7] H. Yu, J. K. Mouw, and V. M. Weaver, Forcing Form and Function: Biomechanical Regulation of Tumor Evolution, Trends Cell Biol. 21, 47 (2011).

[8] A. Mongera, P. Rowghanian, H. J. Gustafson, E. Shelton, D. A. Kealhofer, E. K. Carn, F. Serwane, A. A. Lucio, J. Giammona, and O. Campàs, A Fluid-to-Solid Jamming Transition Underlies Vertebrate Body Axis Elongation, Nature (London) 561, 401 (2018).

[9] B. Wallmeyer, S. Trinschek, S. Yigit, U. Thiele, and T. Betz, Collective Cell Migration in Embryogenesis Follows the Laws of Wetting, Biophys. J. 114, 213 (2018).

[10] X. Trepat and E. Sahai, Mesoscale Physical Principles of Collective Cell Organization, Nat. Phys. 14, 671 (2018).

[11] F. Huber, J. Schnauß, S. Rönicke, P. Rauch, K. Müller, C. Fütterer, and J. Käs, Emergent Complexity of the Cytoskeleton: From Single Filaments to Tissue, Adv. Phys. 62, 1 (2013).

[12] H. T. K. Tse, D. R. Gossett, Y. S. Moon, M. Masaeli, M. Sohsman, Y. Ying, K. Mislick, R. P. Adams, J. Rao, and D. Di Carlo, Quantitative Diagnosis of Malignant Pleural Effusions by Single-Cell Mechanophenotyping, Sci. Transl. Med. 5, 212ra163 (2013).

[13] T. E. Angelini, E. Hannezo, X. Trepat, M. Marquez, J. J. Fredberg, and D. A. Weitz, Glass-like Dynamics of Collective Cell Migration, Proc. Natl. Acad. Sci. U.S.A. 108, 4714 (2011).

[14] K. D. Nnetu, M. Knorr, J. Käs, and M. Zink, The Impact of Jamming on Boundaries of Collectively Moving WeakInteracting Cells, New J. Phys. 14, 115012 (2012).

[15] J.-A. Park et al., Unjamming and Cell Shape in the Asthmatic Airway Epithelium, Nat. Mater. 14, 1040 (2015).

[16] A. Puliafito, L. Hufnagel, P. Neveu, S. Streichan, A. Sigal, D. K. Fygenson, and B. I. Shraiman, Collective and Single Cell Behavior in Epithelial Contact Inhibition, Proc. Natl. Acad. Sci. U.S.A. 109, 739 (2012).

[17] D. Bi, J. H. Lopez, J. M. Schwarz, and M. L. Manning, A Density-Independent Rigidity Transition in Biological Tissues, Nat. Phys. 11, 1074 (2015).
[18] M. Chiang and D. Marenduzzo, Glass Transitions in the Cellular Potts Model, Europhys. Lett. 116, 28009 (2016).

[19] L. Atia, D. Bi, Y. Sharma, J. A. Mitchel, B. Gweon, S. A. Koehler, S. J. DeCamp, B. Lan, J. H. Kim, R. Hirsch, A. F. Pegoraro, K. H. Lee, J. R. Starr, D. A. Weitz, A. C. Martin, J.-A. Park, J. P. Butler, and J. J. Fredberg, Geometric Constraints during Epithelial Jamming, Nat. Phys. 14, 613 (2018).

[20] A. Boromand, A. Signoriello, F. Ye, C. S. O'Hern, and M. D. Shattuck, Jamming of Deformable Polygons, Phys. Rev. Lett. 121, 248003 (2018).

[21] A. J. Liu and S. R. Nagel, The Jamming Transition and the Marginally Jammed Solid, Annu. Rev. Condens. Matter Phys. 1, 347 (2010).

[22] G. Parisi and F. Zamponi, Mean-Field Theory of Hard Sphere Glasses and Jamming, Rev. Mod. Phys. 82, 789 (2010).

[23] D. Bi, J. H. Lopez, J. M. Schwarz, and M. L. Manning, Energy Barriers and Cell Migration in Densely Packed Tissues, Soft Matter 10, 1885 (2014).

[24] G. Duclos, C. Erlenkämper, J.-F. Joanny, and P. Silberzan, Topological Defects in Confined Populations of SpindleShaped Cells, Nat. Phys. 13, 58 (2017).

[25] J. Folkman and A. Moscona, Role of Cell Shape in Growth Control, Nature (London) 273, 345 (1978).

[26] K. Wolf, M. te Lindert, M. Krause, S. Alexander, J. te Riet, A. L. Willis, R. M. Hoffman, C. G. Figdor, S. J. Weiss, and P. Friedl, Physical Limits of Cell Migration: Control by ECM Space and Nuclear Deformation and Tuning by Proteolysis and Traction Force, J. Cell Biol. 201, 1069 (2013).

[27] J. Swift, I. L. Ivanovska, A. Buxboim, T. Harada, P. C. D. P. Dingal, J. Pinter, J. D. Pajerowski, K. R. Spinler, J.-W. Shin, M. Tewari, F. Rehfeldt, D. W. Speicher, and D. E. Discher, Nuclear Lamin-a Scales with Tissue Stiffness and Enhances Matrix-Directed Differentiation, Science 341, 1240104 (2013).

[28] P. Friedl and K. Wolf, Tumour-Cell Invasion and Migration: Diversity and Escape Mechanisms, Nat. Rev. Cancer 3, 362 (2003).

[29] T. M. Koch, S. Münster, N. Bonakdar, J. P. Butler, and B. Fabry, 3d Traction Forces in Cancer Cell Invasion, PLoS One 7, e33476 (2012).

[30] A. Palamidessi, C. Malinverno, E. Frittoli, S. Corallino, E. Barbieri, S. Sigismund, G. V. Beznoussenko, E. Martini, M. Garre, I. Ferrara, C. Tripodo, F. Ascione, E. A. Cavalcanti-Adam, Q. Li, P. P. Di Fiore, D. Parazzoli, F. Giavazzi, R. Cerbino, and G. Scita, Unjamming Overcomes Kinetic and Proliferation Arrest in Terminally Differentiated Cells and Promotes Collective Motility of Carcinoma, Nat. Mater. 18, 1252 (2019).

[31] Y. L. Han, A. F. Pegoraro, H. Li, K. Li, Y. Yuan, G. Xu, Z. Gu, J. Sun, Y. Hao, S. K. Gupta, Y. Li, W. Tang, H. Kang, L. Teng, J. J. Fredberg, and M. Guo, Cell Swelling, Softening and Invasion in a Three-Dimensional Breast Cancer Model, Nat. Phys. 16, 101 (2020).

[32] J. G. Berryman, Random Close Packing of Hard Spheres and Disks, Phys. Rev. A 27, 1053 (1983). 
[33] C. Song, P. Wang, and H. A. Makse, A Phase Diagram for Jammed Matter, Nature (London) 453, 629 (2008).

[34] J. Ranft, M. Basan, J. Elgeti, J.-F. Joanny, J. Prost, and F. Jülicher, Fluidization of Tissues by Cell Division and Apoptosis, Proc. Natl. Acad. Sci. U.S. A. 107, 20863 (2010).

[35] P. Katira, M. H. Zaman, and R. T. Bonnecaze, How Changes in Cell Mechanical Properties Induce Cancerous Behavior, Phys. Rev. Lett. 108, 028103 (2012).

[36] N. Pashayan and P. D. P. Pharoah, The Challenge of Early Detection in Cancer, Science 368, 589 (2020).

[37] R. Staneva, F. El Marjou, J. Barbazan, D. Krndija, S. Richon, A. G. Clark, and D. M. Vignjevic, Cancer Cells in the Tumor Core Exhibit Spatially Coordinated Migration Patterns, J. Cell Sci. 132, jcs220277 (2019).

[38] See Supplemental Material at http://link.aps.org/ supplemental/10.1103/PhysRevX.11.011033 for videos showing the spheroid bulk fusion process, the fluid and arrested movement of nuclei in tumors and cell spheroids, and the 3D segmentation of artificial test data and real 3D spheroids.

[39] J. Schindelin, I. Arganda-Carreras, E. Frise, V. Kaynig, M. Longair, T. Pietzsch, S. Preibisch, C. Rueden, S. Saalfeld, B. Schmid, J.-Y. Tinevez, D. J. White, V. Hartenstein, K. Eliceiri, P. Tomancak, and A. Cardona, Fiji: An OpenSource Platform for Biological-Image Analysis, Nat. Methods 9, 676 (2012), perspective.

[40] S. Pawlizak, A. W. Fritsch, S. Grosser, D. Ahrens, T. Thalheim, S. Riedel, T. R. Kieling, L. Oswald, M. Zink, M. L. Manning, and J. A. Kaes, Testing the Differential Adhesion Hypothesis across the Epithelial-Mesenchymal Transition, New J. Phys. 17, 083049 (2015).

[41] T. Brabletz, R. Kalluri, M. A. Nieto, and R. A. Weinberg, EMT in Cancer, Nat. Rev. Cancer 18, 128 (2018), perspective.

[42] E. Flenner, L. Janosi, B. Barz, A. Neagu, G. Forgacs, and I. Kosztin, Kinetic Monte Carlo and Cellular Particle Dynamics Simulations of Multicellular Systems, Phys. Rev. E 85, 031907 (2012).

[43] J. D. Amack and M. L. Manning, Knowing the Boundaries: Extending the Differential Adhesion Hypothesis in Embryonic Cell Sorting, Science 338, 212 (2012).

[44] P. Chugh and E. K. Paluch, The Actin Cortex at a Glance, J. Cell Sci. 131, jcs186254 (2018).

[45] F. Van Roy and G. Berx, The Cell-Cell Adhesion Molecule E-Cadherin, Cell Mol. Life Sci. 65, 3756 (2008).

[46] A. Donev, I. Cisse, D. Sachs, E. A. Variano, F. H. Stillinger, R. Connelly, S. Torquato, and P. M. Chaikin, Improving the Density of Jammed Disordered Packings Using Ellipsoids, Science 303, 990 (2004).

[47] F. A. Lindemann, Über die Berechnung Molekularer Eigenfrequenzen, Phys. Z. 11, 609 (1910).

[48] J. J. Gilvarry, The Lindemann and Grüneisen Laws, Phys. Rev. 102, 308 (1956).

[49] Z. H. Jin, P. Gumbsch, K. Lu, and E. Ma, Melting Mechanisms at the Limit of Superheating, Phys. Rev. Lett. 87, 055703 (2001).

[50] M. Rueda, C. Ferrer-Costa, T. Meyer, A. Pérez, J. Camps, A. Hospital, J. L. Gelpí, and M. Orozco, A Consensus View of Protein Dynamics, Proc. Natl. Acad. Sci. U.S.A. 104, 796 (2007).
[51] D. Bi, X. Yang, M. C. Marchetti, and M. L. Manning, Motility-Driven Glass and Jamming Transitions in Biological Tissues, Phys. Rev. X 6, 021011 (2016).

[52] M. Merkel and M. L. Manning, A Geometrically Controlled Rigidity Transition in a Model for Confluent 3D Tissues, New J. Phys. 20, 022002 (2018).

[53] S. Hell, G. Reiner, C. Cremer, and E. H. K. Stelzer, Aberrations in Confocal Fluorescence Microscopy Induced by Mismatches in Refractive Index, J. Microsc. 169, 391 (1993).

[54] C. V. Paganelli, A. Olszowka, and A. Ar, The Avian Egg: Surface Area, Volume, and Density, The Condor 76, 319 (1974).

[55] R. Vincent, E. Bazellières, C. Pérez-González, M. Uroz, X. Serra-Picamal, and X. Trepat, Active Tensile Modulus of an Epithelial Monolayer, Phys. Rev. Lett. 115, 248103 (2015).

[56] C. Denais and J. Lammerding, Nuclear Mechanics in Cancer, in Cancer Biology and the Nuclear Envelope: Recent Advances May Elucidate Past Paradoxes, edited by E. C. Schirmer and J. I. de las Heras (Springer, New York, 2014), pp. 435-470.

[57] E. Warmt, S. Grosser, E. Blauth, X. Xie, R. Stange, F. Sauer, J. Schnauß, and K. A. Josef, The Role of Decreasing Cortical Contractility across the EMT in Tissue and Cancer Development (to be published).

[58] C. M. Denais, R. M. Gilbert, P. Isermann, A. L. McGregor, M. Te Lindert, B. Weigelin, P. M. Davidson, P. Friedl, K. Wolf, and J. Lammerding, Nuclear Envelope Rupture and Repair during Cancer Cell Migration, Science 352, 353 (2016).

[59] D. A. Lawson, K. Kessenbrock, R. T. Davis, N. Pervolarakis, and Z. Werb, Tumour Heterogeneity and Metastasis at Single-Cell Resolution, Nat. Cell Biol. 20, 1349 (2018).

[60] O. Ilina, P. G. Gritsenko, S. Syga, J. Lippoldt, C. A. M. La Porta, O. Chepizhko, S. Grosser, M. Vullings, G.-J. Bakker, J. Starru, P. Bult, S. Zapperi, J. A. Kas, A. Deutsch, and P. Friedl, Cell-Cell Adhesion and 3D Matrix Confinement Determine Jamming Transitions in Breast Cancer Invasion, Nat. Cell Biol. 22, 1103 (2020).

[61] A. Saraswathibhatla and J. Notbohm, Tractions and Stress Fibers Control Cell Shape and Rearrangements in Collective Cell Migration, Phys. Rev. X 10, 011016 (2020).

[62] K.-J. Streitberger, L. Lilaj, F. Schrank, J. Braun, K.-T. Hoffmann, M. Reiss-Zimmermann, J. A. Käs, and I. Sack, How Tissue Fluidity Influences Brain Tumor Progression, Proc. Natl. Acad. Sci. U.S.A. 117, 128 (2020).

[63] B. Mathew, A. Schmitz, S. Muñoz-Descalzo, N. Ansari, F. Pampaloni, E. Stelzer, and S. Fischer, Robust and Automated Three-Dimensional Segmentation of Densely Packed Cell Nuclei in Different Biological Specimens with Lines-of-Sight Decomposition, BMC Bioinf. 16, 187 (2015).

[64] U. Schmidt, M. Weigert, C. Broaddus, and G. Myers, Cell Detection with Star-Convex Polygons, in Medical Image Computing and Computer Assisted Intervention-MICCAI 2018. MICCAI 2018., edited by A. Frangi, J. Schnabel, C. Davatzikos, C. Alberola-López, and G. Fichtinger, Lecture 
Notes in Computer Science Vol. 11071 (Springer, Cham, 2018), https://doi.org/10.1007/978-3-030-00934-2_30.

[65] S. G. Krens, J. H. Veldhuis, V. Barone, D. Čapek, J.-L. Maître, G. W. Brodland, and C.-P. Heisenberg, Interstitial Fluid Osmolarity Modulates the Action of Differential Tissue Surface Tension in Progenitor Cell Segregation during Gastrulation, Development 144, 1798 (2017).
[66] N. I. Petridou, S. Grigolon, G. Salbreux, E. Hannezo, and C.-P. Heisenberg, Fluidization-Mediated Tissue Spreading by Mitotic Cell Rounding and Non-canonical WNT Signalling, Nat. Cell Biol. 21, 169 (2019).

[67] L. Sthn, A. Fritschen, J. Choy, M. Dehnert, and C. Dietz, Nanomechanical Sub-surface Mapping of Living Biological Cells by Force Microscopy, Nanoscale 11, 13089 (2019). 